\title{
24. INTERSTITIAL-WATER CHEMISTRY AND DIAGENESIS OF PERIPLATFORM SEDIMENTS FROM THE BAHAMAS, ODP LEG 101'
}

\author{
Peter K. Swart ${ }^{2}$ and Michael Guzikowski²
}

\begin{abstract}
Concentrations of dissolved $\mathrm{Ca}^{2+}, \mathrm{Sr}^{2+}, \mathrm{Mg}^{2+}, \mathrm{SO}_{4}^{2-}$, and alkalinity were measured in pore waters squeezed from sediments taken from ODP Holes 626C and 626D in the Florida Straits; Holes 627A and 627B, 628A, and 630A and 630 north of Little Bahama Bank; Holes 631A, 632A and 632B, and 633A in Exuma Sound; and Holes 634A and $635 \mathrm{~A}$ and 635B in Northeast Providence Channel. These data are compared with the mineralogy and strontium content of the sediments from which the waters were squeezed. Contrasts in the geochemical profiles suggest that significantly different processes govern pore-water signatures at each group of sites. In Little Bahama Bank, strong positive $\mathrm{Ca}^{2+}$ gradients are correlated with weak negative $\mathrm{Mg}^{2+}$ profiles. These trends are analogous to those seen at DSDP sites where carbonate deposits immediately overlie mafic basement, but as the depth to basement may be in excess of $5000 \mathrm{~m}$, we suggest that diffusion gradients are initiated by an underlying sedimentary unit. In contrast, $\mathrm{Ca}^{2+}$ and $\mathrm{Mg}^{2+}$ gradients in Exuma Sound are not developed to any appreciable extent over similar thicknesses of sediment. We suggest that the pore-water chemistry in these deposits is principally controlled by diagenetic reactions occurring within each sequence.

The location and extent of carbonate diagenesis can be estimated from dissolved $\mathrm{Sr}^{2+}$ profiles. In Little Bahama Bank and Exuma Sound, $\mathrm{Sr}^{2+}$ concentrations reach a maximum value of between 700 and $1000 \mu \mathrm{mol} / \mathrm{L}$. Although the depths at which these concentrations are achieved are different for the two areas, the corresponding age of the sediment at the dissolved $\mathrm{Sr}^{2+}$ maximum is similar. Consequently, the diffusive flux of $\mathrm{Sr}^{2+}$ and the calculated rates of recrystallization in the two areas are likewise of a similar magnitude. The rates of recrystallization we calculate are lower than those found in some DSDP pelagic sites. As the waters throughout most of the holes are saturated with respect to $\mathrm{SrSO}_{4}$, celestite precipitation may cause erroneously low $\mathrm{Sr}^{2+}$ production rates and, consequently, low calculated rates of recrystallization. We therefore encourage only the discriminate use of $\mathrm{Sr}^{2+}$ profiles in the quantification of diagenetic processes.
\end{abstract}

\section{INTRODUCTION}

The past decade has seen the emergence of a wealth of data from the Deep Sea Drilling Project (DSDP) concerning the diagenetic behavior of pelagic carbonates buried in the deep marine environment (Schlanger and Douglas, 1974; Lawrence et al., 1975; Matter et al., 1975; Sayles and Mannheim, 1975; Gieskes, 1981; Baker et al., 1982; Elderfield et al., 1982; and others). This research has focused on the post-depositional alteration of carbonate deposits composed primarily of low-magnesium calcite (LMC). Such studies have demonstrated that initial recrystallization of the bulk of the carbonate is predominantly isochemical and occurs within the upper few hundred meters of the sedimentary column. Of particular interest in these sediments has been the development of pore-water dissolved $\mathrm{Sr}^{2+}$ profiles, which typically attain a maximum of about $700 \mu \mathrm{mol} / \mathrm{L}$ (e.g., DSDP Sites 288, 289, 315, and 357; Baker et al., 1982). It has been proposed that the depth at which the $\mathrm{Sr}^{2+}$ maximum is achieved corresponds to the depth of maximum initial recrystallization rate, the profile being maintained by diffusion of liberated $\mathrm{Sr}^{2+}$ ions toward the sediment/water interface above and also perhaps by diffusion into a $\mathrm{Sr}^{2+}$ sink below (Baker, 1981; Baker et al., 1982; Stout, 1985). However, most if not all of this work has been carried out on pelagic carbonate sediments that are dominantly of low-Mg calcitic composition. Not only is this form of calcium carbonate relatively stable, but it also contains only 1000 to $1500 \mathrm{ppm} \mathrm{Sr}$ (Milliman et al., 1974).

\footnotetext{
${ }^{1}$ Austin, J. A., Jr., Schlager, W., et al., Proc. ODP, Sci. Results, 101: College Station, TX (Ocean Drilling Program).

2 Rosenstiel School of Marine and Atmospheric Science-Marine Geology and Geophysics, University of Miami, Miami, FL 33149.
}

In contrast, the sediments surrounding the highly productive, shallow-water platforms of the Bahamas are composed predominantly of metastable carbonates such as aragonite and high$\mathrm{Mg}$ calcite (HMC). Such deposits have been termed periplatform by Schlager and James (1978). In addition to being diagenetically more reactive than LMC, aragonite and HMC often contain much greater concentrations of strontium and magnesium, respectively. For example, coral aragonite typically contains between 7000 and $7500 \mathrm{ppm}$ strontium and $1000 \mathrm{ppm}$ magnesium (Swart, 1981), whereas HMC can have Sr concentrations as high as $3000 \mathrm{ppm}$ and up to $4 \mathrm{wt} \% \mathrm{Mg}$ (Milliman et al., 1974). Consequently, rates of reaction and resultant geochemical profiles might be expected to differ considerably from those of carbonate sites dominated by pelagic sedimentation. It was also expected that the large depth to basement in the Bahamas area would preclude the development of strongly positive $\mathrm{Ca}^{2+}$ gradients such as those observed at DSDP sites where the alteration of underlying oceanic crust is influential. In addition, it was anticipated that the physical isolation of the Bahamas region from noncarbonate terrains would render the deposits there relatively free of "contaminant" terrigenous minerals, thus allowing carbonate dissolution/recrystallization reactions to act as the most important control on pore-fluid chemistry.

\section{ANALYTICAL METHODS}

Pore waters were squeezed from 10-cm-long, whole-round samples on board ship immediately after coring (Austin, Schlager, et al., 1986). Methods and equipment for pore-water extraction were described by Sayles and Mannheim (1975). Derived waters were then titrated for $\mathrm{Cl}^{-}$, $\mathrm{Ca}^{2+}$, and $\mathrm{Mg}^{2+}$ by methods similar to those described by Gieskes $(1973,1974)$. Dissolved $\mathrm{SO}_{4}^{2-}$ was measured using an ion chromatograph. $\mathrm{SO}_{4}^{2-}$ values were then normalized to a surface $\mathrm{SO}_{4}^{2-} / \mathrm{Cl}^{-}$ratio of $(0.0517)$. Even after normalization, the resultant $\mathrm{SO}_{4}^{2-}$ concentrations still showed a high degree of variability. At present, we have no ex- 
planation for these erratic results. Alkalinity and initial $\mathrm{pH}$ were determined using a potentiometric titration method as developed by Dyrssen and Sillen (1967) and others (see Grasshoff, 1976). All additions of acid and calculations were handled on-line by a small laboratory computer (HP-86) interfaced to a $\mathrm{pH}$ meter and autoburette (Metrohm). Water samples needed for onshore analysis were stored in sealed glass ampules. Pore-water $\mathrm{Sr}^{2+}$ concentrations were determined via atomic absorption spectrophotometry at the Rosenstiel School of Marine and Atmospheric Science, University of Miami. Bulk-sediment mineralogies were determined on board ship by X-ray diffraction using the samples that had already been squeezed for interstitial-water samples. In addition, a number of samples on which physical properties had been measured were analyzed. After the cruise, coarse and fine fractions were separated via wet sieving through a $63-\mu \mathrm{m}$ sieve using a buffered sodium borate solution $(\mathrm{pH}=8)$. Samples were then dried at $40^{\circ} \mathrm{C}$. Quantitative mineralogy of the sieved fractions was estimated using the peak area method after analysis on a Philips XRG-3000 X-ray diffractometer at the University of Miami. Strontium concentrations of the sieved solid fractions (coarse and fine) were determined by atomic absorption spectrophotometry after dissolution in $10 \% \mathrm{HNO}_{3}$ and dilution in 1500 $\mu \mathrm{mol} / \mathrm{L} \mathrm{La}, 1 \% \mathrm{HNO}_{3}$ solution.

\section{RESULTS AND DISCUSSION}

The sites cored during Leg 101 are located in four separate areas of the Bahamas: Little Bahama Bank (LBB) sites (627, 628 , and 630), Exuma Sound (ES) sites (631, 632, and 633), Straits of Florida (Site 626), and Northeast Providence Channel sites (634, 635, and 636) (Fig. 1). The latter two areas are grouped under "Channel Sites." For convenience, the results from the mineralogy and interstitial-water analyses will be considered in these three physiographic groups.

\section{Little Bahama Bank}

Holes 627B, 628A, and 630A form a transect from deep to shallower water (drilled in 1025.5, 966, and $807 \mathrm{~m}$ of seawater, respectively) off the northern slope of Little Bahama Bank (LBB). The oldest sediments (late Albian) encountered were in Hole $627 \mathrm{~B}$ at $536 \mathrm{~m}$ sub-bottom. Holes $628 \mathrm{~A}$ and $630 \mathrm{~A}$ reached only 298 and $250 \mathrm{~m}$ sub-bottom, respectively, penetrating sediments of late and middle Miocene age (Austin, Schlager, et al., 1986).

\section{Mineralogy}

Surface sediments at all LBB sites consist of a combination of HMC, aragonite, LMC, and, except at Hole 628A, terrige-

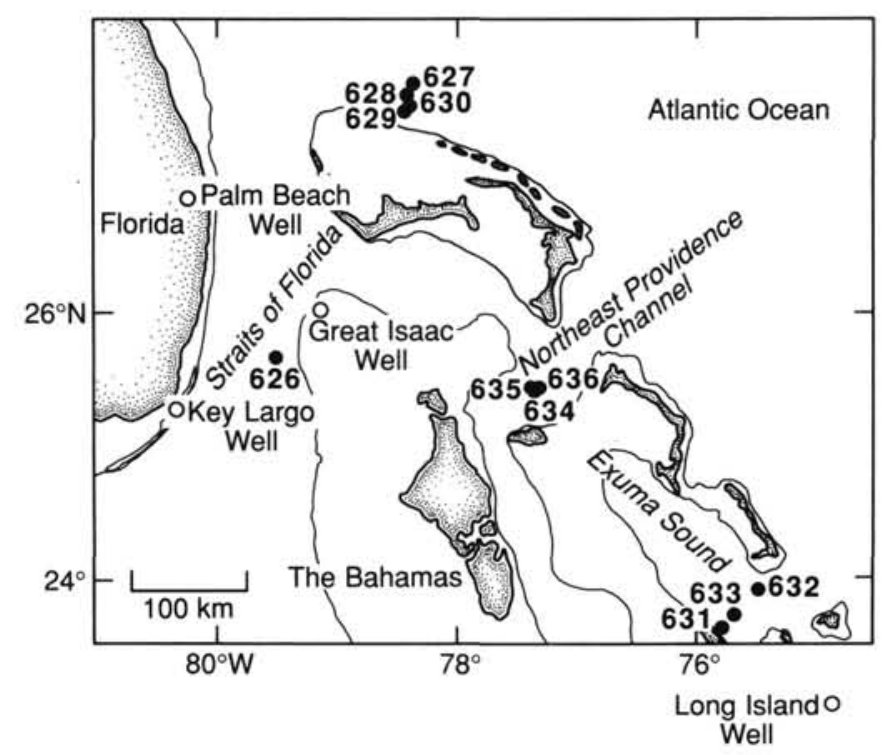

Figure 1. Location map of the sites drilled during Leg 101. nous minerals (Table 1). The HMC component is entirely absent below $10 \mathrm{~m}$ sub-bottom, perhaps reflecting its relative instability in the deep-sea diagenetic environment (Austin, Schlager, et al., 1986). The aragonitic component also seems to recrystallize quite rapidly, as only minor amounts of aragonite were found below $150 \mathrm{~m}$ sub-bottom; the two samples showing aragonite below this depth in Hole 627B are attributed to downhole contamination. Minor amounts of quartz, feldspars, and clay minerals were detected in many of the X-rayed samples (Austin, Schlager, et al., 1986). The change in the dominant mineralogy from aragonite to LMC can be attributed both to dissolution/recrystallization of the aragonitic component and to a downhole change from bank-margin sedimentation to more open-ocean conditions.

A terrigenous unit was encountered between 350 and $470 \mathrm{~m}$ sub-bottom in Hole 627B. The principal minerals present within this unit are quartz, microcline, albite, and sanidine, with minor quantities of palygorskite and sepiolite. Whereas studies by Zemmels et al. (1972) and Droxler (1984) identified the feldspars as plagioclase, our data indicate that these feldspars have a composition closer to albite than to anorthite (Fig. 2); therefore, they do not contain a large amount of calcium. This unit is interpreted (Austin, Schlager, et al., 1986) as a terrigenous shelf deposit of Cenomanian age (100 Ma). The large concentration of these minerals in an otherwise predominantly carbonate terrain is unexpected and indicates that during Cenomanian time conditions were suitable for the input of clastic material from a nearby felsic terrain.

The Cenomanian marl is underlain by a carbonate/evaporite unit of late Albian age. This basal section consists primarily of calcite, dolomite, and gypsum, and has been interpreted as a partially dolomitized, shallow carbonate platform deposit (Austin, Schlager, et al., 1986).

\section{Interstitial-Water Chemistry}

All three LBB holes (and Hole 630C) are characterized by strong positive $\mathrm{Ca}^{2+}$ gradients and weak negative $\mathrm{Mg}^{2+}$ gradients, the most pronounced being at Site 627 and the least pronounced at Sites 628 and 630 (Table 2 and Figs. 3 through 6). Although no bottom-water samples were collected, the dissolved $\mathrm{Mg}^{2+}$ gradients appear to be steepest in the upper part of the deposits. Even after normalization to the dissolved $\mathrm{Cl}^{-}$concentration, the uppermost pore-water samples show their steepest dissolved $\mathrm{Mg}^{2+}$ gradients within the upper $15 \mathrm{~m}$ of the section. Interstitial $\mathrm{Sr}^{2+}$ concentrations reach maximum values of 600 to $700 \mu \mathrm{mol} / \mathrm{L}$ at depths of 30,60 , and $110 \mathrm{~m}$ sub-bottom in Holes $627 \mathrm{~B}, 628 \mathrm{~A}$, and 630A, respectively (Figs. 3 through 5). However, the age of the sediment in which this is achieved is similar in all instances (approximately $5 \mathrm{Ma}$ ) (Austin, Schlager, et al., 1986; Guzikowski et al., 1986). The concentration of $\mathrm{Sr}^{2+}$ is maintained throughout the depth of Holes $628 \mathrm{~A}$ and $630 \mathrm{~A}$, whereas in Hole 627B (the deepest hole) there is a slight depletion toward the bottom of the hole. Dissolved $\mathrm{SO}_{4}^{2-}$ concentrations remain at, or slightly below, seawater values throughout all three holes, with the exception of Hole $627 \mathrm{~B}$, in which there is an actual increase to $34 \mathrm{mmol} / \mathrm{L}$ at $437.4 \mathrm{~m}$ sub-bottom. This rise in $\mathrm{SO}_{4}^{2-}$ is attributable to an increase in salinity as the $\mathrm{SO}_{4}^{2-} / \mathrm{Cl}^{-}$ratio remains constant with depth. The absence of significant amounts of sulfate reduction is reflected in the alkalinity values, which are only slightly elevated relative to surface seawater, indicating that little oxidation of organic matter is now occurring (Table 2).

The presence of inversely related $\mathrm{Ca}^{2+}$ and $\mathrm{Mg}^{2+}$ gradients (Fig. 7) is a surprising feature of the LBB pore-water analyses, as no proximal basaltic basement or significant volcanic component exists within the deposits to induce such gradients. However, sediments recovered from Hole 627B define two units that may act as sources for $\mathrm{Ca}^{2+}$ ions and sinks for the $\mathrm{Mg}^{2+}$ ions, 
Table 1. Bulk sediment mineralogy, Little Bahama Bank sites.

\begin{tabular}{|c|c|c|c|c|}
\hline $\begin{array}{l}\text { Depth } \\
\text { (mbsf) }\end{array}$ & $\begin{array}{c}\text { Calcite } \\
(\%)\end{array}$ & $\begin{array}{c}\text { Aragonite } \\
(\%)\end{array}$ & $\begin{array}{c}\text { Dolomite } \\
(\%)\end{array}$ & $\begin{array}{c}\text { Quartz } \\
(\%)\end{array}$ \\
\hline \multicolumn{5}{|l|}{ Hole 627B } \\
\hline 4.4 & 53 & 0 & 31 & 7 \\
\hline 6.7 & 75 & 25 & 0 & 0 \\
\hline 13.2 & 51 & 44 & 1 & 4 \\
\hline 22.9 & 64 & 32 & 3 & 0 \\
\hline 32.6 & 79 & 18 & 2 & 0 \\
\hline 42.1 & 93 & 0 & 7 & 0 \\
\hline 46.4 & 89 & 6 & 5 & 0 \\
\hline 47.4 & 83 & 7 & 10 & 0 \\
\hline 54.1 & 93 & 0 & 4 & 3 \\
\hline 56.0 & 95 & 0 & 2 & 3 \\
\hline 60.5 & 100 & 0 & 0 & 0 \\
\hline 60.5 & 100 & 0 & 0 & 0 \\
\hline 61.2 & 77 & 16 & 7 & 0 \\
\hline 64.0 & 62 & 35 & 3 & 0 \\
\hline 78.5 & 100 & 0 & 0 & 0 \\
\hline 78.5 & 100 & 0 & 0 & 0 \\
\hline 79.6 & 100 & 0 & 0 & 0 \\
\hline 79.7 & 88 & 12 & 0 & 0 \\
\hline 80.3 & 98 & 0 & 0 & 2 \\
\hline 90.7 & 59 & 41 & 0 & 0 \\
\hline 90.8 & 66 & 31 & 0 & 3 \\
\hline 90.9 & 65 & 32 & 0 & 3 \\
\hline 99.4 & 53 & 47 & 0 & 0 \\
\hline 109.0 & 98 & 0 & 0 & 2 \\
\hline 117.0 & 100 & 0 & 0 & 0 \\
\hline 119.5 & 99 & 0 & 0 & 1 \\
\hline 123.0 & 99 & 0 & 0 & 1 \\
\hline 123.6 & 96 & 0 & 0 & 4 \\
\hline 130.6 & 86 & 14 & 0 & 0 \\
\hline 136.2 & 98 & 0 & 0 & 2 \\
\hline 145.1 & 79 & 21 & 0 & 0 \\
\hline 154.1 & 98 & 0 & 0 & 2 \\
\hline 162.9 & 98 & 0 & 0 & 2 \\
\hline 177.0 & 99 & 0 & 1 & 0 \\
\hline 182.3 & 100 & 0 & 0 & 0 \\
\hline 198.4 & 82 & 16 & 2 & 0 \\
\hline 200.9 & 99 & 0 & 0 & 1 \\
\hline 216.1 & 85 & 16 & 2 & 0 \\
\hline 239.8 & 98 & 0 & 0 & 2 \\
\hline 256.1 & 98 & 0 & 0 & 2 \\
\hline 258.2 & 98 & 0 & 0 & 2 \\
\hline 260.4 & 100 & 0 & 0 & 0 \\
\hline 281.0 & 100 & 0 & 0 & 0 \\
\hline 282.7 & 100 & 0 & 0 & 0 \\
\hline 290.0 & 100 & 0 & 0 & 0 \\
\hline 298.2 & 100 & 0 & 0 & 0 \\
\hline 306.3 & 100 & 0 & 0 & 0 \\
\hline 317.2 & 100 & 0 & 0 & 0 \\
\hline 334.0 & 100 & 0 & 0 & 0 \\
\hline 345.0 & 100 & 0 & 0 & 0 \\
\hline 362.0 & 65 & 0 & 2 & 33 \\
\hline 363.1 & 88 & 0 & 0 & 12 \\
\hline 366.4 & 59 & 0 & 0 & 41 \\
\hline 380.9 & 70 & 0 & 2 & 28 \\
\hline 384.6 & 76 & 0 & 2 & 22 \\
\hline 399.3 & 73 & 0 & 4 & 23 \\
\hline 403.7 & 90 & 0 & 2 & 8 \\
\hline 416.1 & 93 & 0 & 1 & 6 \\
\hline 421.5 & 91 & 0 & 1 & 8 \\
\hline 437.4 & 88 & 0 & 2 & 10 \\
\hline 487.1 & 0 & 0 & 100 & 0 \\
\hline 519.2 & 100 & 0 & 0 & 0 \\
\hline 524.2 & 0 & 0 & 0 & 0 \\
\hline 527.8 & 0 & 0 & 0 & 0 \\
\hline
\end{tabular}

the Cenomanian marl and the upper Albian evaporites. Although the terrigenous platform deposit of Cenomanian age was first believed to be responsible for generating the necessary $\mathrm{Ca}^{2+}$ gradients (Austin, Schlager, et al., 1986), subsequent X-ray study has shown that no calcium-bearing igneous minerals are present in this unit (see Fig. 2). Nevertheless, alteration of the feldspars to $\mathrm{Mg}$-bearing clay minerals such as sepiolite and palygorskite
Table 1 (continued).

\begin{tabular}{rrrrr}
\hline $\begin{array}{c}\text { Depth } \\
(\mathrm{mbsf})\end{array}$ & $\begin{array}{c}\text { Calcite } \\
(\%)\end{array}$ & $\begin{array}{c}\text { Aragonite } \\
(\%)\end{array}$ & $\begin{array}{c}\text { Dolomite } \\
(\%)\end{array}$ & $\begin{array}{c}\text { Quartz } \\
(\%)\end{array}$ \\
\hline Hole 628A & & & & \\
& & & & \\
3.0 & 49 & 48 & 3 & 0 \\
8.9 & 36 & 62 & 2 & 0 \\
9.0 & 27 & 69 & 3 & 0 \\
9.8 & 33 & 41 & 15 & 11 \\
10.0 & 26 & 71 & 3 & 0 \\
11.0 & 42 & 55 & 3 & 0 \\
18.6 & 42 & 58 & 0 & 0 \\
28.8 & 78 & 20 & 2 & 0 \\
40.0 & 88 & 0 & 12 & 0 \\
57.6 & 82 & 13 & 4 & 2 \\
59.9 & 89 & 8 & 2 & 1 \\
78.0 & 79 & 13 & 7 & 0 \\
96.5 & 87 & 13 & 0 & 0 \\
110.0 & 69 & 31 & 0 & 0 \\
124.8 & 84 & 16 & 0 & 0 \\
148.3 & 96 & 0 & 3 & 1 \\
153.5 & 99 & 0 & 0 & 1 \\
167.3 & 94 & 0 & 0 & 6 \\
190.6 & 99 & 0 & 0 & 1 \\
209.7 & 99 & 0 & 0 & 1 \\
238.4 & 100 & 0 & 0 & 0 \\
267.6 & 99 & 0 & 0 & 1 \\
288.8 & 99 & 0 & 0 & 1
\end{tabular}

Hole $630 \mathrm{~A}$

$\begin{array}{rrrrl}0.70 & 21 & 76 & 4 & 0 \\ 2.20 & 77 & 27 & 0 & 0 \\ 2.22 & 41 & 59 & 0 & 0 \\ 3.50 & 83 & 7 & 10 & 0 \\ 7.40 & 45 & 55 & 0 & 0 \\ 8.10 & 50 & 50 & 0 & 0 \\ 16.00 & 51 & 45 & 4 & 0 \\ 18.20 & 28 & 46 & 18 & 0 \\ 25.60 & 48 & 48 & 2 & 0 \\ 35.20 & 52 & 42 & 6 & 0 \\ 44.80 & 74 & 24 & 2 & 0 \\ 64.30 & 77 & 22 & 2 & 0 \\ 83.30 & 80 & 18 & 2 & 0 \\ 105.10 & 94 & 0 & 6 & 0 \\ 140.90 & 88 & 0 & 12 & 0 \\ 169.80 & 83 & 11 & 6 & 0 \\ 200.60 & 78 & 11 & 11 & 0 \\ 224.80 & 83 & 14 & 3 & 0\end{array}$

Hole 630B

$\begin{array}{lllll}3.04 & 64 & 36 & 0 & 0 \\ 3.34 & 47 & 51 & 2 & 0\end{array}$

Hole $630 \mathrm{C}$

\begin{tabular}{lllll}
1.45 & 23 & 75 & 3 & 0 \\
2.95 & 56 & 44 & 0 & 0 \\
4.45 & 21 & 75 & 4 & 0 \\
5.95 & 66 & 34 & 0 & 0 \\
7.45 & 58 & 42 & 0 & 0 \\
8.95 & 42 & 57 & 1 & 0 \\
\hline
\end{tabular}

may be responsible for the slight depletions in both dissolved $\mathrm{Mg}^{2+}$ and $\mathrm{Sr}^{2+}$ concentrations evident at the bottom of Hole 627B (Gieskes, 1981).

A second possible source of $\mathrm{Ca}^{2+}$ ions is the evaporite sequence that underlies the Cenomanian marl. This unit, late $\mathrm{Al}$ bian in age, was first encountered at a depth of $478 \mathrm{~m}$ sub-bottom in Hole 627B (Austin, Schlager, et al., 1986). The dissolution of gypsum and the subsequent diffusion of $\mathrm{Ca}^{2+}$ ions toward the sediment/water interface is an attractive alternative as the underlying control on Little Bahama Bank $\mathrm{Ca}^{2+}$ gradients. Although no pore waters were recovered from the evaporite unit, fluids throughout the core are undersaturated with respect to gypsum. In addition, the generally high $\mathrm{SO}_{4}^{2-}$ values through- 


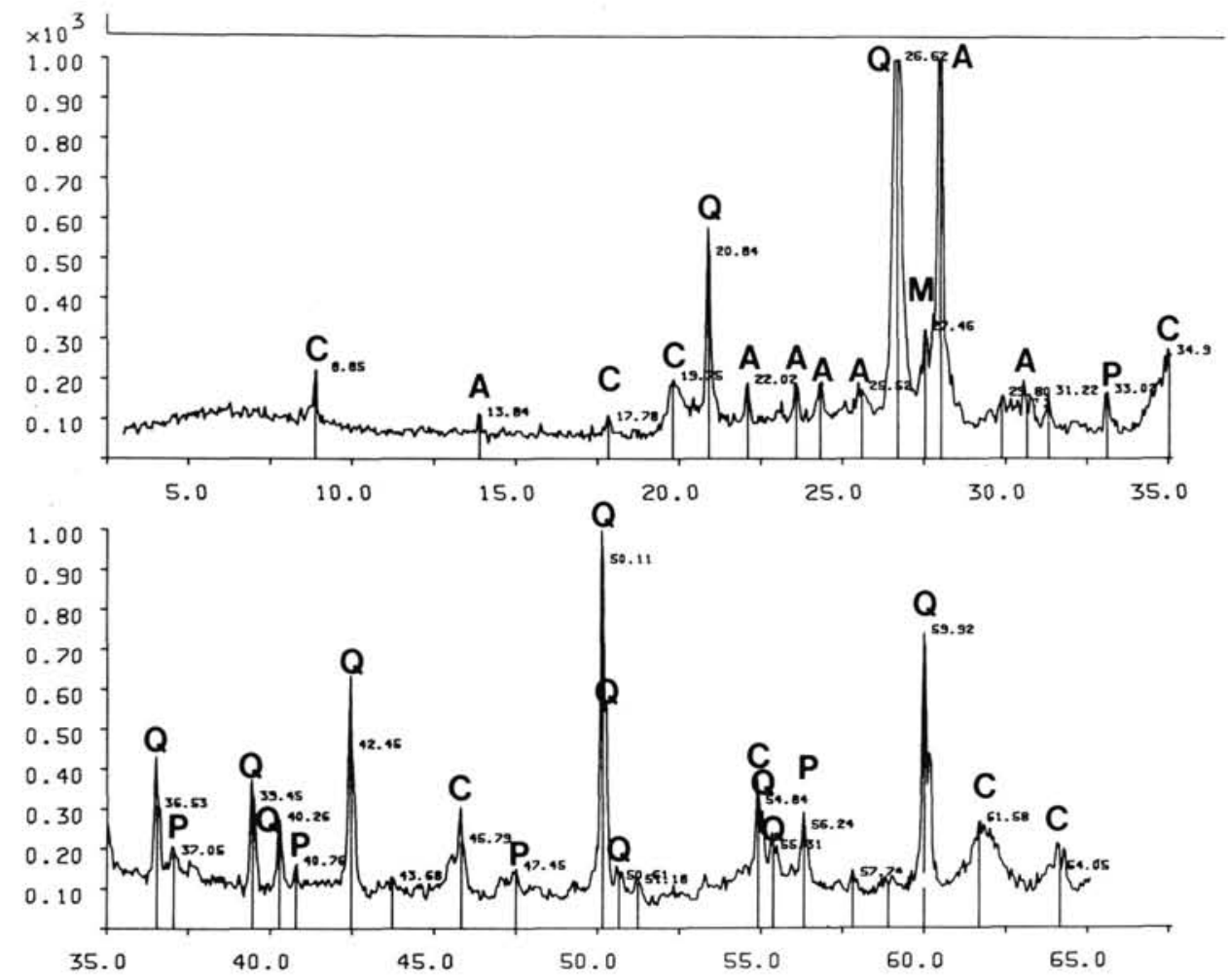

Figure 2. X-ray diffractogram of insoluble material isolated from Sample 101-627B-38X-5, 115-117 cm. Peaks are marked as $A$, albite; $C$, clay minerals; $M$, microcline; $P$, pyrite; $Q$, quartz. Peak positions of albite indicate low calcium concentration.

out the core and a shift toward even higher concentrations at the bottom of the hole support this theory. It is interesting to note that the increase in $\mathrm{SO}_{4}^{2-}$ is directly correlated with a depletion in dissolved $\mathrm{Sr}^{2+}$ values, which indicates that the precipitation of celestite may be limiting the maximum concentrations of these two species. Our calculations show that pore waters throughout most of the cores are approximately saturated with respect to this mineral. This may also explain why there is not a one-to-one increase in dissolved $\mathrm{Ca}^{2+}$ and $\mathrm{SO}_{4}^{2-}$ concentrations associated with the postulated dissolution of gypsum.

The LBB sites all show steep dissolved $\mathrm{Ca}^{2+}$ and $\mathrm{Mg}^{2+}$ gradients in the shallow subsurface. To investigate these gradients in more detail, pore-water samples were squeezed on board ship from every section of a 10-m core taken at Site 630 (Hole 630C) (Fig. 6; Table 2). These data show a progressive increase in the $\mathrm{Ca}^{2+}$ concentration to a depth of $8.95 \mathrm{~m}$ sub-bottom. The $\mathrm{Mg}^{2+}$ concentration, however, abruptly decreases between the surface and the first sample squeezed. Although this may be an artifact of squeezing, when normalized to the $\mathrm{Cl}^{-}$so as to remove the influences of the high surface salinity, a slight decrease remains, indicating an actual net consumption of $\mathrm{Mg}^{2+}$. We believe that the gradients in the upper $10 \mathrm{~m}$ of all three LBB sites are related to carbonate dissolution and precipitation reactions. In particular, the simultaneous occurrence of HMC dissolution (which is virtually absent below $10 \mathrm{~m}$ sub-bottom) and the precipitation of dolomite would impart $\mathrm{Ca}^{2+}$ ions to, and remove $\mathrm{Mg}^{2+}$ ions from, the local pore waters.

\section{Exuma Sound}

The Exuma Sound (ES) sites also lie on a deep- to shallowwater transect: Holes 632A and 632B (1996 m water depth), Hole 633A (1681 m water depth), and Hole 631A (1081 m water depth) (Austin, Schlager, et al., 1986). As a result of being surrounded by highly productive shallow-water platforms, the Exuma Sound sites have average sedimentation rates that are somewhat higher than those calculated for the sites north of Little Bahama Bank (Austin, Schlager, et al., 1986; Watkins et al., this volume); hence, the oldest sediments drilled here were only middle Miocene in age.

\section{Mineralogy}

Surface sediments at the Exuma Sound sites are composed of LMC, HMC, aragonite, and small amounts of dolomite and clay minerals (Table 3). Surprisingly, aragonite is the dominant mineral throughout the entire cored interval of the Exuma Sound deposits. Holes 631A and 632A do, however, display decreasing aragonite contents in their lowest sections. Although these decreases may be related to variations in the original input signal, the dissolution of aragonite and HMC and the concomitant precipitation of LMC and dolomite may also be important. In addition, celestite $\left(\mathrm{SrSO}_{4}\right)$ was detected in samples recovered from depths of 58 and $150 \mathrm{~m}$ sub-bottom in Hole 632A.

\section{Interstitial-Water Chemistry}

In contrast to the Little Bahama Bank sites, the chemical profiles at Exuma Sound generally show only small changes in dissolved $\mathrm{Ca}^{2+}$ concentrations with depth (Figs. 8 through 10). Holes 632A, 632B, and 633A show slight depletions in $\mathrm{Ca}^{2+}$ downhole, whereas Hole $631 \mathrm{~A}$ displays an increase from 10 to $17 \mathrm{mmol} / \mathrm{L}$ between 66 and $184 \mathrm{~m}$ sub-bottom.

At Holes $632 \mathrm{~A}$ and $632 \mathrm{~B}, \mathrm{Mg}^{2+}$ concentration changes little throughout the cores, whereas the other two Exuma Sound sites display significant $\mathbf{M g}^{2+}$ depletions at depth. The depleted samples from Hole $631 \mathrm{~A}$ correspond to the waters found to be enriched in $\mathrm{Ca}^{2+}$.

Dissolved $\mathrm{Sr}^{2+}$ concentrations for the Exuma Sound sites all show an increase to magnitudes of about $700 \mu \mathrm{mol} / \mathrm{L}$ within the 
Table 2. Interstitial-water data, Leg 101.

\begin{tabular}{|c|c|c|c|c|c|c|c|c|c|}
\hline $\begin{array}{l}\text { Core/ } \\
\text { section }\end{array}$ & $\begin{array}{l}\text { Depth } \\
\text { (mbsf) }\end{array}$ & $\mathrm{pH}$ & $\underset{(\mathrm{mmol} / \mathrm{L})}{\mathrm{Ca}^{2+}}$ & $\underset{(\mathrm{mmol} / \mathrm{L})}{\mathrm{Mg}^{2+}}$ & $\underset{(\mu \mathrm{mol} / \mathrm{L})}{\mathrm{Sr}^{2+}}$ & $\begin{array}{l}\text { Alkalinity } \\
\text { (meq/L) }\end{array}$ & $\underset{(\mathrm{mmol} / \mathrm{L})}{\mathrm{SO}_{4}^{2-}}$ & $\begin{array}{l}\mathrm{Cl}^{-} \\
(\mathrm{ppt})\end{array}$ & $\underset{(p p t)}{S}$ \\
\hline \multicolumn{10}{|l|}{ Hole $626 \mathrm{C}$} \\
\hline S.S. & - & 8.20 & 10.27 & 52.53 & - & 2.25 & 28.51 & 19.11 & 35.5 \\
\hline $3-X-3$ & 20.9 & 7.83 & 10.13 & 50.99 & 115 & 1.81 & 27.55 & 19.41 & 36.4 \\
\hline $14-\mathrm{H}-5$ & 129.1 & 7.44 & 14.40 & 52.10 & 595 & 3.36 & 26.15 & 19.84 & 37.0 \\
\hline $15-\mathrm{H}-5$ & 138.7 & 7.38 & 16.22 & 45.46 & 606 & 3.28 & 26.13 & 20.13 & 37.5 \\
\hline $18-\mathrm{H}-5$ & 167.4 & 7.33 & 14.25 & 52.19 & 610 & 3.62 & 28.33 & 20.66 & 38.0 \\
\hline \multicolumn{10}{|l|}{ Hole 627B } \\
\hline S.S. & - & 8.26 & 10.42 & 54.68 & - & 2.21 & 28.90 & 20.54 & - \\
\hline $1-\mathrm{H}-3$ & 4.4 & 7.64 & 11.12 & 52.31 & 279 & 3.08 & 28.90 & 19.37 & 33.9 \\
\hline $2-\mathrm{H}-5$ & 13.2 & 7.72 & 11.28 & 49.81 & 433 & 3.51 & 26.62 & 18.67 & 35.6 \\
\hline $3-\mathrm{H}-5$ & 22.9 & 7.57 & 11.74 & 49.07 & 600 & 4.08 & 27.97 & 19.52 & 35.6 \\
\hline 4-H-5 & 32.6 & 7.41 & 12.62 & 48.58 & 611 & 3.51 & 26.17 & 18.95 & 35.5 \\
\hline $5-\mathrm{H}-5$ & 42.1 & 7.03 & 12.89 & 50.09 & 561 & 3.23 & 26.11 & 18.67 & 34.0 \\
\hline 7-H-5 & 61.2 & 7.32 & 12.68 & 49.75 & 633 & 3.53 & 27.43 & 21.25 & 35.5 \\
\hline 9-H-5 & 80.3 & 7.43 & 14.31 & 48.56 & 627 & 4.06 & 32.38 & 20.05 & 35.6 \\
\hline $11-\mathrm{H}-5$ & 99.4 & 7.36 & 15.86 & 47.29 & 660 & 4.00 & 27.47 & 19.76 & 34.5 \\
\hline $12-\mathrm{H}-5$ & 108.9 & 7.42 & 15.52 & 47.52 & 635 & 3.97 & 27.92 & 20.07 & 35.9 \\
\hline $15-\mathrm{H}-4$ & 136.2 & 7.15 & 16.44 & 48.44 & 625 & 3.80 & 24.10 & 20.61 & 35.9 \\
\hline $17-X-3$ & 154.1 & 7.43 & 17.49 & 48.72 & 582 & 3.91 & 25.80 & 19.27 & 36.0 \\
\hline $21-X-3$ & 198.4 & 7.77 & 10.74 & 52.13 & 179 & 1.71 & 26.25 & 21.04 & 36.5 \\
\hline $23-X-4$ & 216.1 & 7.74 & 11.12 & 51.75 & 164 & 2.16 & 23.50 & 20.93 & 37.0 \\
\hline $27-X-5$ & 256.1 & 7.16 & 23.78 & 70.94 & 548 & 3.30 & 25.23 & 20.37 & 37.2 \\
\hline $30-X-3$ & 281.0 & 7.22 & 16.69 & 60.09 & 545 & 3.13 & 29.24 & 21.25 & 37.8 \\
\hline $33-X-1$ & 307.0 & 7.15 & 25.32 & 46.46 & 506 & 2.69 & 27.56 & 20.79 & 37.0 \\
\hline $42-X-5$ & 399.3 & 7.36 & 30.30 & 39.25 & 392 & 2.58 & 24.60 & 20.56 & 37.8 \\
\hline $46-X-5$ & 437.4 & 7.66 & 35.29 & 44.83 & - & 2.61 & 34.03 & 23.45 & 40.2 \\
\hline \multicolumn{10}{|l|}{ Hole $628 \mathrm{~A}$} \\
\hline S.S. & - & 8.23 & 10.70 & 54.50 & - & 2.34 & - & 17.73 & 36.1 \\
\hline $1-\mathrm{H}-1$ & 1.4 & 7.80 & 10.47 & 52.60 & 162 & 3.01 & 27.54 & 18.46 & 35.0 \\
\hline 2-H-5 & 11.0 & 7.74 & 11.53 & 52.90 & 376 & 3.51 & 25.95 & 19.65 & 35.0 \\
\hline 3-H-5 & 20.6 & 7.61 & 11.64 & 51.55 & 495 & 4.05 & 26.24 & 19.00 & 35.0 \\
\hline 4-H-5 & 30.3 & 7.47 & 12.67 & 50.72 & 596 & 3.77 & 25.41 & 18.59 & 35.0 \\
\hline 5-H-5 & 40.0 & 7.42 & 12.84 & 51.70 & 634 & 5.22 & 26.40 & 20.02 & 35.1 \\
\hline 7-H-5 & 58.9 & 7.54 & 14.13 & 48.73 & 670 & 4.02 & 26.40 & 20.14 & 35.2 \\
\hline $9-\mathrm{H}-6$ & 79.5 & 7.39 & 14.58 & 47.49 & 677 & 4.52 & 27.81 & 19.81 & 35.6 \\
\hline $11-\mathrm{H}-5$ & 96.5 & 7.36 & 12.96 & 50.01 & 670 & 4.19 & 27.20 & 19.27 & 35.9 \\
\hline 14-H-5 & 124.8 & 7.40 & 15.41 & 47.17 & 686 & 4.30 & 26.77 & 19.88 & 35.9 \\
\hline $17-\mathrm{H}-5$ & 153.5 & 7.39 & 16.09 & 45.87 & 669 & 3.94 & 28.16 & 19.95 & 36.0 \\
\hline $21-\mathrm{H}-2$ & 184.7 & 7.41 & 16.35 & 47.18 & 721 & 3.85 & 24.98 & 20.46 & 36.2 \\
\hline $23-\mathrm{H}-5$ & 209.7 & 7.40 & 16.26 & 48.39 & 655 & 3.27 & 24.18 & 19.04 & 36.0 \\
\hline $26-X-1$ & 232.8 & 7.43 & 17.89 & 45.81 & 502 & 3.21 & 22.80 & 18.97 & 36.0 \\
\hline $29-X-1$ & 261.6 & 7.39 & 19.27 & 45.49 & 568 & 4.02 & 24.23 & 20.21 & 37.0 \\
\hline \multicolumn{10}{|l|}{ Hole $630 \mathrm{~A}$} \\
\hline S.S. & - & 8.21 & 10.43 & 58.00 & - & 2.41 & 31.40 & 21.05 & 36.2 \\
\hline $1-\mathrm{H}-5$ & 7.4 & 7.65 & 11.54 & 52.87 & 254 & 3.00 & 26.68 & 20.81 & 35.0 \\
\hline 2-H-5 & 16.0 & 7.67 & 12.03 & 52.45 & 296 & 2.90 & 30.84 & 20.28 & 35.2 \\
\hline $3-\mathrm{H}-5$ & 25.6 & 7.72 & 11.78 & 52.65 & 300 & 3.09 & 30.98 & 19.27 & 35.1 \\
\hline 4-H-5 & 35.2 & 7.80 & 11.57 & 54.39 & 273 & 3.29 & 30.39 & 20.18 & 35.0 \\
\hline $5-\mathrm{H}-5$ & 44.8 & 7.63 & 12.25 & 53.12 & 300 & 3.08 & 30.69 & 20.56 & 35.1 \\
\hline 7-H-5 & 64.3 & 7.55 & 12.52 & 52.82 & 440 & 2.99 & 30.65 & 20.07 & 35.7 \\
\hline $9-\mathrm{H}-5$ & 83.3 & 7.35 & 14.30 & 50.71 & 550 & 3.41 & 29.17 & 20.84 & 35.9 \\
\hline $12-\mathrm{H}-5$ & 112.1 & 7.47 & 14.97 & 49.81 & 585 & 3.80 & 28.68 & 20.63 & 36.2 \\
\hline $15-\mathrm{H}-5$ & 140.9 & 7.36 & 14.64 & 48.96 & 611 & 4.05 & 29.28 & 21.16 & 37.0 \\
\hline 18-H-5 & 169.8 & 7.43 & 16.58 & 50.55 & 618 & 4.22 & 30.97 & 21.82 & 37.5 \\
\hline $21-X-5$ & 200.6 & 7.51 & 16.20 & 50.93 & 648 & 4.69 & 27.36 & 21.58 & 37.8 \\
\hline $24-X-2$ & 224.8 & 7.52 & 17.07 & 51.36 & 630 & 5.07 & 29.09 & 21.33 & 39.6 \\
\hline \multicolumn{10}{|l|}{ Hole $630 \mathrm{C}$} \\
\hline $1-\mathrm{H}-1$ & 1.45 & 7.75 & 10.74 & 53.92 & 148 & 3.03 & 30.34 & 19.72 & 34.8 \\
\hline 1-H-2 & 2.95 & 7.70 & 10.85 & 53.72 & 164 & 2.87 & 36.39 & 19.34 & 34.8 \\
\hline $1-\mathrm{H}-3$ & 4.45 & 7.62 & 10.78 & 53.85 & 187 & 2.78 & 24.76 & 20.00 & 34.8 \\
\hline 1-H-4 & 5.95 & 7.62 & 10.97 & 53.51 & 206 & 2.90 & 33.81 & 20.72 & 35.0 \\
\hline 1-H-5 & 7.45 & 7.65 & 11.47 & 51.94 & 234 & 3.08 & 34.09 & 20.14 & 35.0 \\
\hline $1-\mathrm{H}-6$ & 8.95 & 7.61 & 11.50 & 52.69 & 255 & 2.85 & 35.07 & 20.49 & 35.2 \\
\hline
\end{tabular}

upper $100 \mathrm{~m}$ of the sediment column. Although the depths at which these concentrations are achieved are somewhat greater than those at the Little Bahama Bank sites, the age of the sediment at these depths is similar for the two areas (Austin, Schlager, et al., 1986; Guzikowski et al., 1986). In Hole 633A, the dissolved $\mathrm{Sr}^{2+}$ concentrations peak at over $1000 \mu \mathrm{mol} / \mathrm{L}$ in the deepest samples (Fig. 10).

Alkalinity values at all Exuma Sound sites show an increase with depth, reaching levels as high as $22 \mathrm{mmol} / \mathrm{L}$ in Hole $633 \mathrm{~A}$ (Fig. 10). Dissolved $\mathrm{SO}_{4}^{2-}$ profiles of Holes 631A and 632A and 
Table 2 (continued).

\begin{tabular}{|c|c|c|c|c|c|c|c|c|c|}
\hline $\begin{array}{l}\text { Core/ } \\
\text { section }\end{array}$ & $\begin{array}{l}\text { Depth } \\
\text { (mbsf) }\end{array}$ & $\mathrm{pH}$ & $\underset{(\mathrm{mmol} / \mathrm{L})}{\mathrm{Ca}^{2+}}$ & $\underset{(\mathrm{mmol} / \mathrm{L})}{\mathrm{Mg}^{2+}}$ & $\underset{(\mu \mathrm{mol} / \mathrm{L})}{\mathrm{Sr}^{2+}}$ & $\begin{array}{c}\text { Alkalinity } \\
\text { (meq/L) }\end{array}$ & $\underset{(\mathrm{mmol} / \mathrm{L})}{\mathrm{SO}_{4}^{2-}}$ & $\begin{array}{l}\mathrm{Cl}^{-} \\
\text {(ppt) }\end{array}$ & $\underset{(\mathrm{ppt})}{\mathrm{S}}$ \\
\hline \multicolumn{10}{|l|}{ Hole 631A } \\
\hline S.S. & - & 8.28 & 10.66 & 58.91 & 102 & 2.45 & 30.63 & 20.53 & 36.2 \\
\hline $1-\mathrm{H}-5$ & 7.4 & 7.72 & 10.70 & 54.68 & 150 & 3.30 & 28.27 & 19.81 & 35.0 \\
\hline $2-\mathrm{H}-5$ & 17.1 & 7.79 & 10.78 & 54.96 & 187 & 2.99 & 27.22 & 19.44 & 34.8 \\
\hline $3-\mathrm{H}-5$ & 27.0 & 7.67 & 10.51 & 54.63 & 177 & 2.98 & 26.96 & 19.75 & 34.8 \\
\hline $4-\mathrm{H}-5$ & 36.9 & 7.64 & 11.32 & 54.06 & 212 & 3.00 & 31.83 & 19.71 & 35.0 \\
\hline 5-H-5 & 46.5 & 7.64 & 11.58 & 54.28 & 308 & 4.65 & 26.81 & 19.41 & 35.2 \\
\hline 7-H-5 & 65.6 & 7.71 & 9.97 & 57.68 & 463 & 6.90 & 25.47 & 20.22 & 35.9 \\
\hline $10-\mathrm{H}-5$ & 91.5 & 7.52 & 10.36 & 57.89 & 651 & 10.18 & 26.33 & 20.29 & 36.2 \\
\hline $13-X-5$ & 126.7 & 7.41 & 11.51 & 56.14 & 747 & 12.02 & 28.63 & 20.05 & 37.8 \\
\hline $16-X-1$ & 149.4 & 7.66 & 15.25 & 52.88 & 753 & 13.77 & 29.19 & 21.07 & 37.0 \\
\hline $19-X-5$ & 184.4 & 7.90 & 16.84 & 52.13 & 753 & 10.09 & 29.33 & 20.46 & 36.8 \\
\hline \multicolumn{10}{|l|}{ Hole $632 \mathrm{~A}$} \\
\hline S.S. & - & 8.21 & 10.93 & 56.67 & 105 & 2.42 & 29.90 & 20.83 & 36.2 \\
\hline $1-\mathrm{H}-4$ & 5.9 & 7.73 & 9.94 & 54.72 & 211 & 3.16 & 24.59 & 19.52 & 34.8 \\
\hline $2-\mathrm{H}-5$ & 14.3 & 7.74 & 8.72 & 50.41 & 242 & 3.47 & 21.16 & 19.48 & 35.2 \\
\hline $3-\mathrm{H}-4$ & 22.6 & 7.18 & 9.60 & 55.06 & 271 & 3.62 & 25.57 & 18.72 & 34.8 \\
\hline $4-\mathrm{H}-5$ & 33.7 & 7.51 & 8.80 & 55.04 & 360 & 4.11 & 21.75 & 18.00 & 35.5 \\
\hline $5-\mathrm{H}-5$ & 43.3 & 7.60 & 9.90 & 56.82 & 233 & 2.38 & 23.62 & 19.55 & 37.0 \\
\hline $8-\mathrm{H}-5$ & 72.0 & 7.42 & 11.01 & 54.94 & 600 & 5.37 & 25.43 & 19.96 & 35.8 \\
\hline $12-\mathrm{H}-5$ & 101.3 & 7.52 & 11.77 & 55.24 & 724 & 5.44 & 30.33 & 19.17 & 35.4 \\
\hline \multicolumn{10}{|l|}{ Hole 632B } \\
\hline $10-R-2$ & 210.4 & 7.91 & 10.59 & 57.00 & 283 & 2.43 & 29.57 & 19.82 & 36.2 \\
\hline \multicolumn{10}{|l|}{ Hole $633 \mathrm{~A}$} \\
\hline S.S. & - & 8.14 & 10.73 & 56.73 & 101 & 2.43 & 28.89 & 19.36 & 36.0 \\
\hline $1-\mathrm{H}-5$ & 7.4 & 7.63 & 9.92 & 53.15 & 263 & 3.45 & 26.88 & 19.74 & 34.8 \\
\hline $2-\mathrm{H}-5$ & 16.1 & 7.51 & 9.65 & 54.30 & 265 & 3.68 & 25.62 & 19.44 & 34.8 \\
\hline $3-\mathrm{H}-5$ & 25.7 & 7.52 & 9.84 & 55.87 & 298 & 4.05 & 25.68 & 19.27 & 34.8 \\
\hline 4-H-4 & 35.4 & 7.45 & 9.73 & 55.16 & 382 & 5.16 & 25.34 & 19.74 & 35.2 \\
\hline $5-\mathrm{H}-5$ & 45.0 & 7.67 & 9.99 & 53.02 & 428 & 6.51 & 26.05 & 19.98 & 35.8 \\
\hline 7-H-5 & 64.3 & 7.52 & 9.82 & 52.96 & 565 & 9.76 & 24.82 & 19.81 & 36.2 \\
\hline $13-X-5$ & 120.1 & 7.21 & 8.72 & 42.31 & 1008 & 19.78 & 18.70 & 21.06 & 37.4 \\
\hline $16-X-5$ & 149.1 & 7.31 & 9.39 & 41.42 & 1001 & 22.44 & 16.85 & 21.80 & 38.0 \\
\hline $23-X-3$ & 212.9 & 7.86 & 10.66 & 53.88 & 169 & 2.53 & 27.65 & 19.36 & 36.4 \\
\hline \multicolumn{10}{|l|}{ Hole $634 \mathrm{~A}$} \\
\hline S.S. & - & 8.10 & 10.91 & 55.47 & 101 & 2.43 & 31.21 & 19.52 & 35.6 \\
\hline $1-R-4$ & 5.9 & 7.60 & 10.51 & 51.73 & 175 & 3.56 & 26.85 & 18.61 & 35.0 \\
\hline $2-R-4$ & 149.9 & 7.54 & 11.57 & 51.24 & 171 & 3.00 & 27.30 & 19.72 & 35.4 \\
\hline $4-R-2$ & 166.0 & 7.68 & 11.57 & 51.13 & 143 & 2.94 & 26.68 & 19.27 & 35.4 \\
\hline
\end{tabular}

Note: S.S. = surface seawater.

632B show a zone of depleted $\mathrm{SO}_{4}^{2-}$ values in the upper $100 \mathrm{~m}$ of the section (Figs. 8 and 9). $\mathrm{SO}_{4}^{2-}$ concentrations of the waters from Hole $633 \mathrm{~A}$ progressively decrease throughout the core to a value of under $17 \mathrm{mmol} / \mathrm{L}$ at $150 \mathrm{~m}$ sub-bottom.

The slight enrichment in $\mathrm{Ca}^{2+}$ and depletion in $\mathrm{Mg}^{2+}$ at depth in Hole 631A may be a result of dolomite precipitation in the lower portion of the cored section. An increase in the proportion of dolomite downhole supports this theory (Tables 3 and 6). The negative $\mathrm{Mg}^{2+}$ gradient, depleted $\mathrm{SO}_{4}^{2-}$ levels, and high alkalinity concentrations in Hole $633 \mathrm{~A}$ are probably also associated with a zone of active dolomitization (see section on dolomitization). The samples most depleted in dissolved $\mathrm{Mg}^{2+}$ correspond to maximum pore-water alkalinity values (alkalinity $>$ $22 \mathrm{mmol} / \mathrm{L}$ ). The existence of depleted $\mathrm{SO}_{4}^{2-}$ levels in this zone indicates that the high alkalinities are induced by the degradation of buried organic matter via sulfate reduction. We believe that the precipitation of dolomite, induced by the high alkalinity levels, resulted in consumption of $\mathrm{Mg}^{2+}$ ions.

In Figure 11, we have plotted the $\mathrm{SrSO}_{4}$ ion molar products for the pore fluids of Holes $627 \mathrm{~B}$ and $633 \mathrm{~A}$. Note that the vertical line in this diagram is only our best estimate of the celestite precipitation boundary (i.e., $\mathrm{K}^{*}{ }_{\mathrm{Sp}}$ ) (Culberson et al., 1978; Reardon and Armstrong, 1987). This diagram does not account for the variations in the $\mathrm{K}^{*}$ sp of $\mathrm{SrSO}_{4}$ owing to downhole changes in physical conditions (e.g., temperature, ionic strength, and so on). Nevertheless, it is significant that the pore waters are generally at or near saturation throughout most of the cores. Although the concentrations of dissolved $\mathrm{Sr}^{2+}$ are low relative to dissolved $\mathrm{SO}_{4}^{2-}$, the amount of strontium initially present in the solids is capable, upon release to the pore fluids, of removing large quantities of $\mathrm{SO}_{4}^{2-}$ via celestite precipitation. Therefore, celestite dissolution/precipitation may be important not only in checking the concentrations of both $\mathrm{SO}_{4}^{2-}$ and $\mathrm{Sr}^{2+}$ but also in determining the overall shape of the profiles for these species. This has significant implications for the reliability of dissolved $\mathrm{Sr}^{2+}$ profiles as tools for quantitative description of carbonate diagenesis. Where celestite is actively precipitating, dissolved $\mathrm{Sr}^{2+}$ levels will be limited to lower values than would otherwise be attainable. Similarly, the dissolution of celestite in zones with undersaturated pore waters will artificially enhance dissolved $\mathrm{Sr}^{2+}$ and $\mathrm{SO}_{4}^{2-}$ concentrations.

Despite the higher concentrations of $\mathrm{Sr}^{2+}$ in Hole 633A, the calculated solubility products of $\mathrm{SrSO}_{4}$ for the pore fluids are similar to those in Hole 627B. It may be that the dissolution and precipitation of celestite control the $\mathrm{Sr}^{2+}$ concentration of the pore fluids. Hence, the depleted $\mathrm{SO}_{4}^{2-}$ levels at depth in Hole 


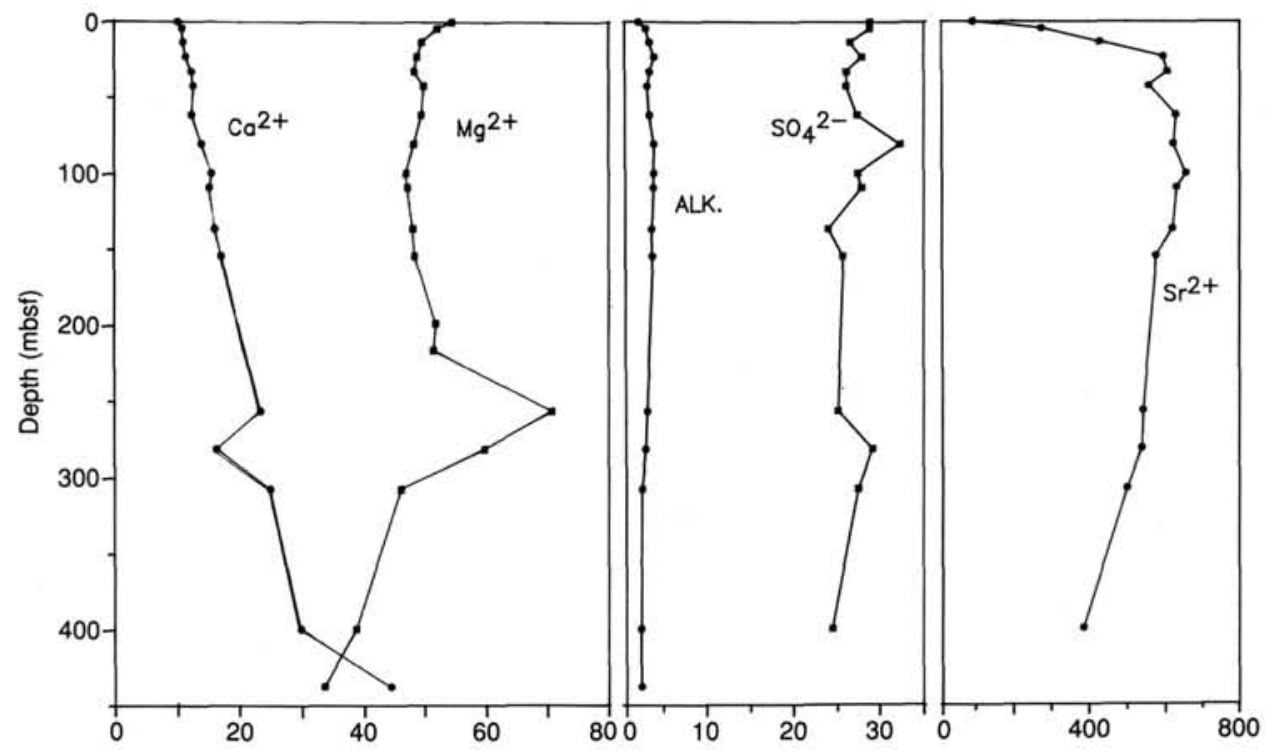

Figure 3. Interstitial pore-water chemistry, Hole 627B. Units of value for the various mineral constituents are as follows: $\mathrm{Ca}^{2+}, \mathrm{mmol} / \mathrm{L} ; \mathrm{Mg}^{2+}, \mathrm{mmol} / \mathrm{L}$; alkalinity, $\mathrm{mmol} / \mathrm{L} ; \mathrm{SO}_{4}^{2-}, \mathrm{mmol} / \mathrm{L} ; \mathrm{Sr}^{2+}, \mu \mathrm{mol} / \mathrm{L}$.

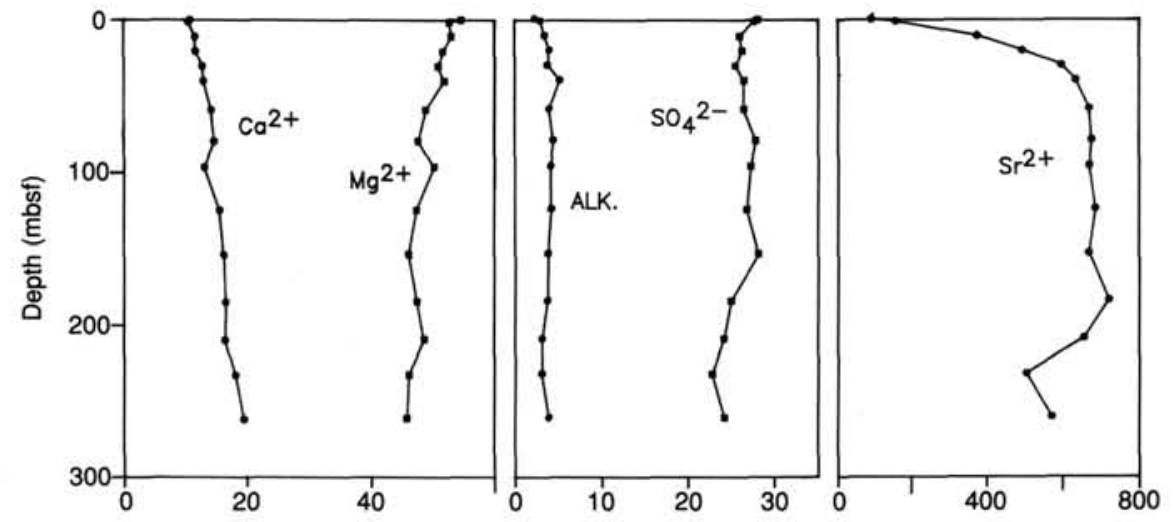

Figure 4. Interstitial pore-water chemistry, Hole 628A. See Figure 3 caption for units of value.

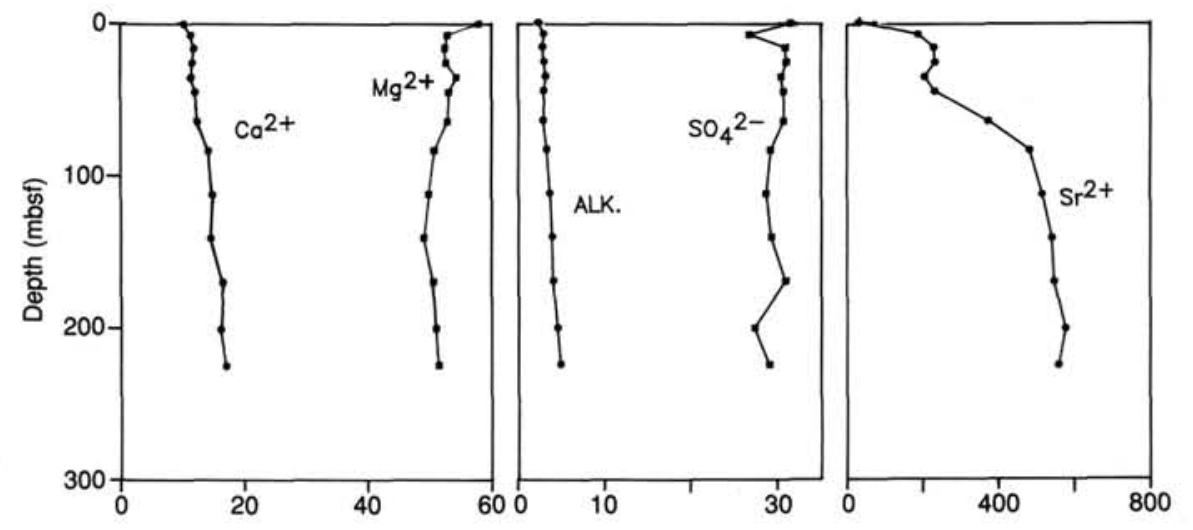

Figure 5. Interstitial pore-water chemistry, Hole 630A. See Figure 3 caption for units of value. 


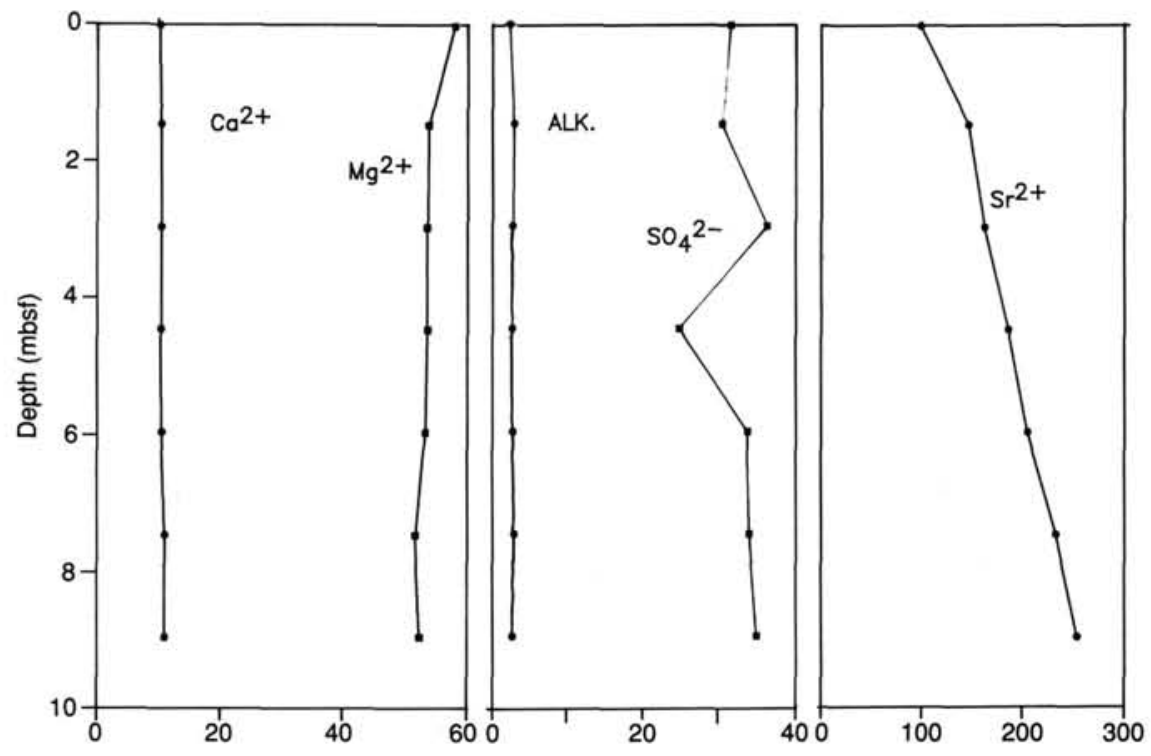

Figure 6. Interstitial pore-water chemistry, Hole $630 \mathrm{C}$. See Figure 3 caption for units of value.

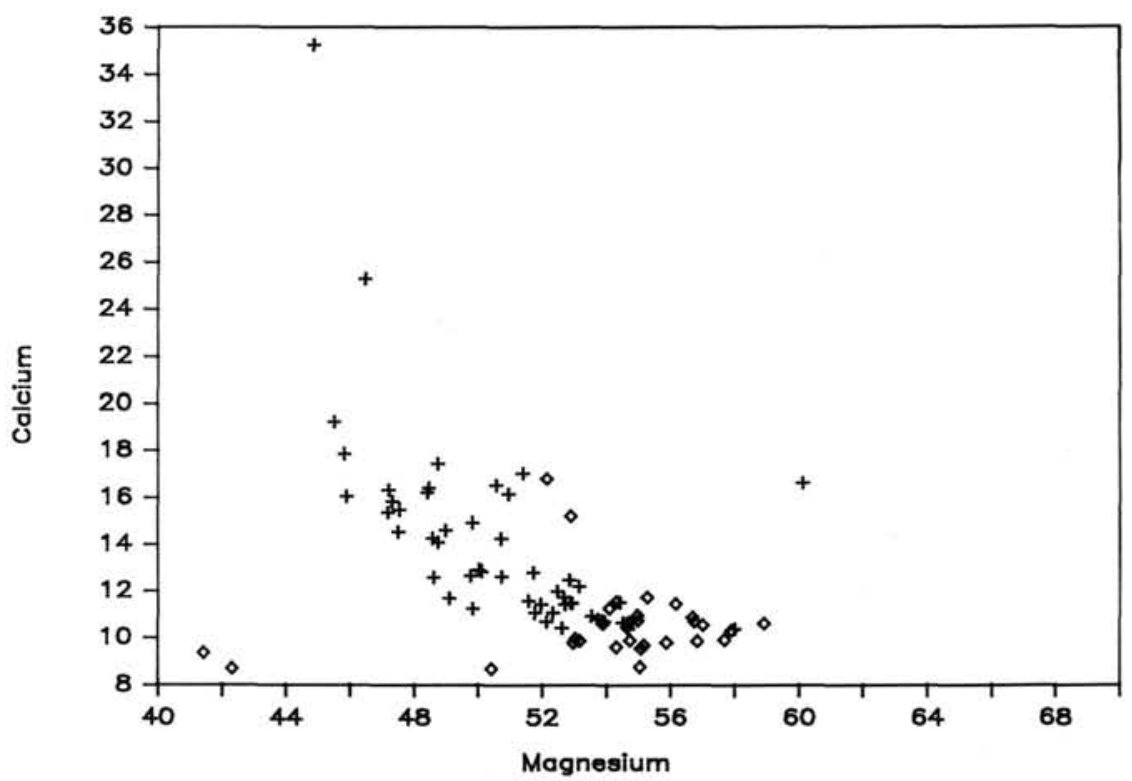

Figure 7. Pore-water concentrations of $\mathrm{Ca}^{2+}$ and $\mathrm{Mg}^{2+}$ (in $\mathrm{mmol} / \mathrm{L}$ ) at Little Bahama Bank (pluses) and Exuma Sound (diamonds) sites. Note the difference in association of the two elements in these two areas.

633A allow the dissolved $\mathrm{Sr}^{2+}$ levels to rise without exceeding the solubility constant of $\mathrm{SrSO}_{4}$ (celestite).

\section{Channel Sites}

In addition to the Little Bahama Bank and Exuma Sound transects, holes were drilled in the Straits of Florida (Site 626) and in Northeast Providence Channel (Sites 634, 635, and 636). As a result of differing objectives at these sites, the recovered material was less than ideal for interstitial-water studies (Table 2).

\section{Mineralogy}

Samples from Holes $626 \mathrm{C}$ and $626 \mathrm{D}$ revealed that aragonite and $\mathrm{HMC}$ were present erratically throughout, probably as a consequence of downhole contamination. The sediments were composed of LMC in addition to a small background concen- tration of dolomite (Tables 4 and 6). In Hole 634A, surface sediments consisted of mixtures of LMC, HMC, aragonite, quartz, and minor amounts of microcline and clay minerals. Below $1.9 \mathrm{~m}$ sub-bottom, aragonite disappears, and the sediment is composed of LMC and small quantities of dolomite and quartz. A similar pattern was found at Holes 635A and 635B, except that aragonite persisted to a depth of $23 \mathrm{~m}$ sub-bottom. In addition, this site was characterized by an abundance of diagenetic iron sulfides such as marcasite and pyrite (Austin, Schlager, et al., 1986).

\section{Interstitial-Water Chemistry}

Only four water samples could be taken from Hole $626 \mathrm{C}$ in addition to the surface-seawater sample (Table 2 ). These analyses revealed the presence of a positive $\mathrm{Ca}^{2+}$ gradient similar in 
Table 3. Bulk sediment mineralogy, Exuma Sound sites.

\begin{tabular}{rcccc}
\hline $\begin{array}{c}\text { Depth } \\
(\mathrm{mbsf})\end{array}$ & $\begin{array}{c}\text { Calcite } \\
(\%)\end{array}$ & $\begin{array}{c}\text { Aragonite } \\
(\%)\end{array}$ & $\begin{array}{c}\text { Dolomite } \\
(\%)\end{array}$ & $\begin{array}{c}\text { Quartz } \\
(\%)\end{array}$ \\
\hline Hole 631A & & & & \\
& & & & \\
7.4 & 28 & 70 & 2 & 0 \\
17.1 & 41 & 56 & 3 & 0 \\
27.0 & 47 & 51 & 2 & 0 \\
36.8 & 28 & 69 & 3 & 0 \\
47.5 & 39 & 61 & 0 & 0 \\
65.6 & 32 & 66 & 0 & 0 \\
70.2 & 39 & 56 & 4 & 0 \\
81.2 & 37 & 56 & 6 & 0 \\
89.4 & 18 & 70 & 12 & 0 \\
94.6 & 25 & 68 & 7 & 0 \\
124.4 & 14 & 71 & 15 & 0 \\
126.6 & 17 & 69 & 14 & 0 \\
131.0 & 20 & 68 & 12 & 0 \\
137.0 & 24 & 66 & 10 & 0 \\
149.3 & 22 & 66 & 12 & 0 \\
159.6 & 32 & 60 & 8 & 0 \\
184.3 & 25 & 65 & 10 & 0 \\
206.2 & 51 & 42 & 6 & 0 \\
236.1 & 71 & 19 & 10 & 0
\end{tabular}

Holes 632A and 632B

\begin{tabular}{|c|c|c|c|c|}
\hline 5.9 & 36 & 61 & 3 & 0 \\
\hline 13.3 & 16 & 80 & 3 & 0 \\
\hline 24.1 & 59 & 40 & 0 & 2 \\
\hline 33.7 & 14 & 86 & 0 & 0 \\
\hline 43.3 & 19 & 78 & 3 & 0 \\
\hline 50.6 & 45 & 50 & 5 & 0 \\
\hline 53.6 & 59 & 41 & 0 & 0 \\
\hline 57.2 & 27 & 59 & 14 & 0 \\
\hline 70.2 & 75 & 22 & 3 & 0 \\
\hline 72.0 & 38 & 55 & 7 & 0 \\
\hline 76.4 & 41 & 48 & 11 & 0 \\
\hline 84.5 & 32 & 64 & 4 & 0 \\
\hline 93.9 & 58 & 36 & 5 & 0 \\
\hline 104.9 & 43 & 53 & 4 & 0 \\
\hline 110.1 & 58 & 38 & 4 & 0 \\
\hline 112.7 & 81 & 18 & 2 & 0 \\
\hline 130.8 & 56 & 37 & 7 & 0 \\
\hline 140.7 & 65 & 23 & 12 & 0 \\
\hline 149.6 & 0 & 0 & 0 & 0 \\
\hline 149.6 & 58 & 39 & 3 & 0 \\
\hline 159.2 & 65 & 31 & 4 & 0 \\
\hline 168.9 & 70 & 26 & 4 & 0 \\
\hline 169.2 & 54 & 41 & 5 & 0 \\
\hline 178.7 & 44 & 51 & 5 & 0 \\
\hline 179.3 & 75 & 14 & 11 & 0 \\
\hline 188.6 & 61 & 32 & 7 & 0 \\
\hline 209.5 & 42 & 53 & 5 & 0 \\
\hline 227.4 & 75 & 15 & 9 & 0 \\
\hline 236.9 & 76 & 15 & 8 & 0 \\
\hline 237.1 & 72 & 17 & 11 & 0 \\
\hline 255.5 & 63 & 31 & 6 & 0 \\
\hline 258.6 & 65 & 30 & 4 & 0 \\
\hline 260.0 & 80 & 15 & 4 & 0 \\
\hline
\end{tabular}

magnitude to those discovered at the Little Bahama Bank sites, but no $\mathrm{Mg}^{2+}$ gradient. $\mathrm{SO}_{4}^{2-}$ concentrations remain high, and alkalinity is only slightly elevated relative to that of surface seawater.

Three water samples taken from Hole 634A showed little change compared with surface seawater. However, there was an increase in $\mathrm{Ca}^{2+}$ from 10.5 to $11.6 \mathrm{mmol} / \mathrm{L}$ between 5.9 and $166.0 \mathrm{~m}$ sub-bottom, which may suggest the presence of an underlying $\mathrm{Ca}^{2+}$ source.

\section{Sediment Diagenesis}

\section{Estimates of Recrystallization}

Biogenically precipitated carbonates generally have higher strontium concentrations than their inorganic counterparts (Katz
Table 3 (continued).

\begin{tabular}{|c|c|c|c|c|}
\hline $\begin{array}{l}\text { Depth } \\
\text { (mbsf) }\end{array}$ & $\begin{array}{c}\text { Calcite } \\
(\%)\end{array}$ & $\begin{array}{l}\text { Aragonite } \\
(\%)\end{array}$ & $\begin{array}{c}\text { Dolomite } \\
(\%)\end{array}$ & $\begin{array}{c}\text { Quartz } \\
(\%)\end{array}$ \\
\hline \multicolumn{5}{|l|}{ Hole 633A } \\
\hline 2.2 & 25 & 72 & 3 & 0 \\
\hline 4.2 & 38 & 57 & 3 & 2 \\
\hline 7.4 & 59 & 41 & 0 & 0 \\
\hline 8.2 & 61 & 39 & 0 & 0 \\
\hline 10.9 & 85 & 13 & 0 & 1 \\
\hline 16.1 & 74 & 26 & 0 & 0 \\
\hline 20.5 & 70 & 23 & 3 & 3 \\
\hline 23.5 & 55 & 43 & 2 & 0 \\
\hline 25.7 & 47 & 53 & 0 & 0 \\
\hline 26.5 & 26 & 72 & 2 & 0 \\
\hline 28.0 & 57 & 43 & 0 & 0 \\
\hline 33.2 & 42 & 58 & 0 & 0 \\
\hline 36.1 & 64 & 36 & 0 & 0 \\
\hline 39.8 & 98 & 2 & 0 & 0 \\
\hline 45.0 & 86 & 14 & 0 & 0 \\
\hline 45.8 & 48 & 38 & 14 & 0 \\
\hline 49.4 & 42 & 54 & 4 & 0 \\
\hline 55.4 & 11 & 70 & 19 & 0 \\
\hline 59.1 & 28 & 70 & 2 & 0 \\
\hline 64.3 & 6 & 75 & 19 & 0 \\
\hline 68.6 & 15 & 75 & 10 & 0 \\
\hline 93.2 & 35 & 53 & 12 & 0 \\
\hline 120.1 & 41 & 49 & 10 & 0 \\
\hline 123.9 & 33 & 57 & 10 & 0 \\
\hline 124.5 & 26 & 64 & 10 & 0 \\
\hline 135.4 & 28 & 62 & 10 & 0 \\
\hline 143.9 & 69 & 15 & 16 & 0 \\
\hline 149.1 & 66 & 30 & 4 & 0 \\
\hline 151.5 & 51 & 47 & 3 & 0 \\
\hline 160.3 & 43 & 20 & 40 & 0 \\
\hline 169.7 & 19 & 71 & 10 & 0 \\
\hline 190.7 & 31 & 58 & 11 & 0 \\
\hline 212.6 & 37 & 58 & 5 & 0 \\
\hline 217.8 & 37 & 58 & 5 & 0 \\
\hline
\end{tabular}

et al., 1972; Baker et al., 1982). Consequently, there is a net release of $\mathrm{Sr}^{2+}$ ions to the pore waters associated with the initial recrystallization of biogenic sediments to inorganic calcite and dolomite (Baker et al., 1982; Stout, 1985). For this reason, dissolved $\mathrm{Sr}^{2+}$ profiles have often been used to examine the dynamics of sediment diagenesis within deep-sea carbonate deposits (e.g., Baker et al., 1982; Stout, 1985; Baker, 1986). These studies indicate that recrystallization generally begins soon after deposition but that the overall rates at which diagenesis proceeds are highly variable from site to site. Factors affecting recrystallization rate include initial sediment composition, local geothermal gradient, and sedimentation rate (Berner, 1980).

One method involves integration of the $\mathrm{Sr}^{2+}$ flux for the time period during which it has been operating (Baker, 1981; Stout, 1985). Diffusive flux of $\mathrm{Sr}^{2+}$ can be calculated from Fick's Law:

$$
\text { Flux }=-\mathrm{D}_{\mathrm{b}}(\delta c / \delta z)
$$

where

$$
\begin{aligned}
\mathrm{D}_{\mathrm{b}} & =\text { bulk-sediment diffusion coefficient; } \\
\delta c / \delta z & =\text { concentration gradient over depth, } z
\end{aligned}
$$

Using this equation, we have calculated the $\mathrm{Sr}^{2+}$ fluxes for the upper sections of the Little Bahama Bank and Exuma Sound sites (Table 5). In these calculations, we have assumed a linear concentration gradient for dissolved $\mathrm{Sr}^{2+}$ and a constant bulk sediment diffusion coefficient for $\mathrm{Sr}^{2+}$ of $\left(3.5 \times 10^{-6} \mathrm{~mol} /\right.$ $\mathrm{cm}^{2} / \mathrm{s}$ ). The appropriate depth (z) corresponds to the depth over which the $\mathrm{Sr}^{2+}$ gradient is significantly positive. The total amount of $\mathrm{Sr}^{2+}$ that these fluxes would generate $\left(\mathrm{\Sigma Sr}^{2+}\right)$ over their respective time periods can then be calculated by multiplying the 

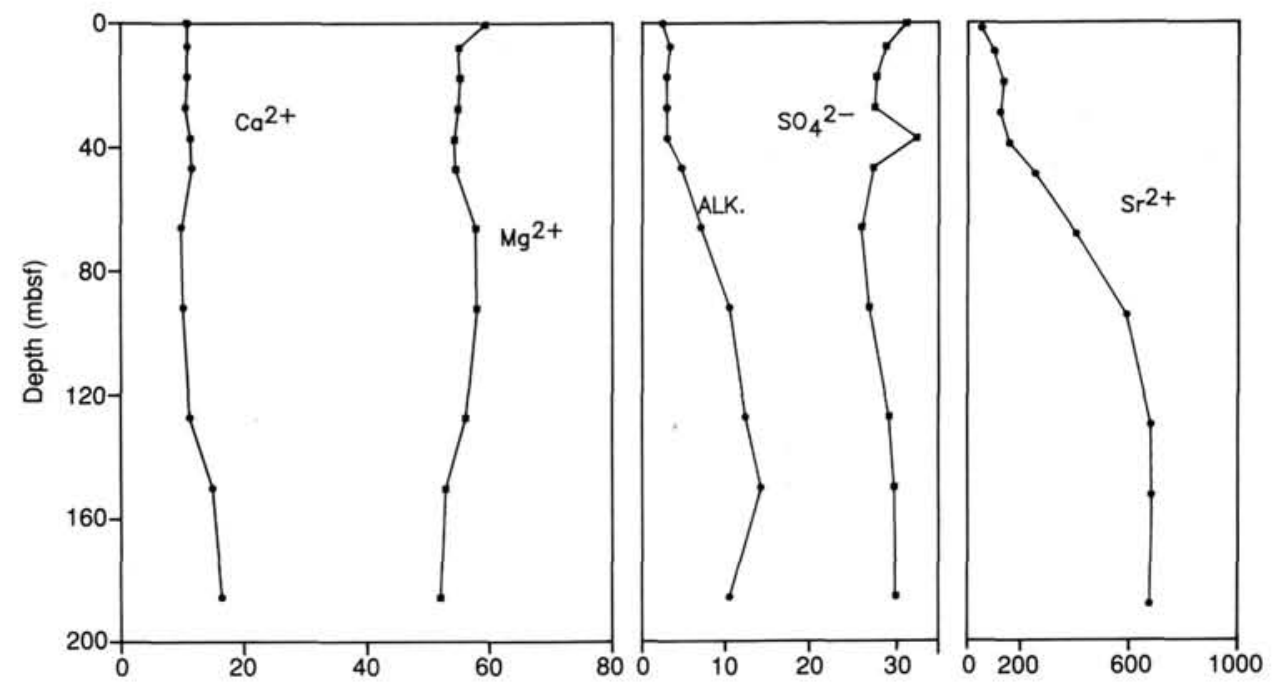

Figure 8. Interstitial pore-water chemistry, Hole 631A. See Figure 3 caption for units of value.
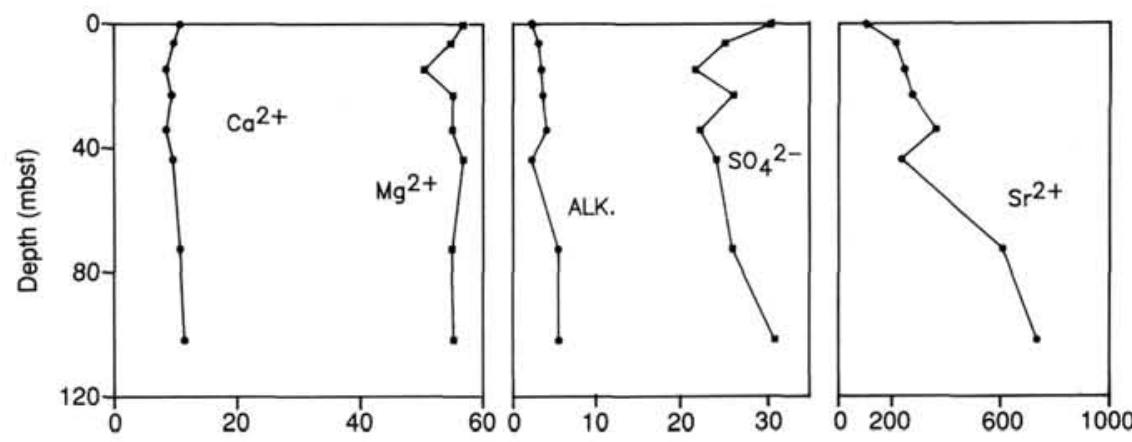

Figure 9. Interstitial pore-water chemistry, Hole 632A. See Figure 3 caption for units of value.
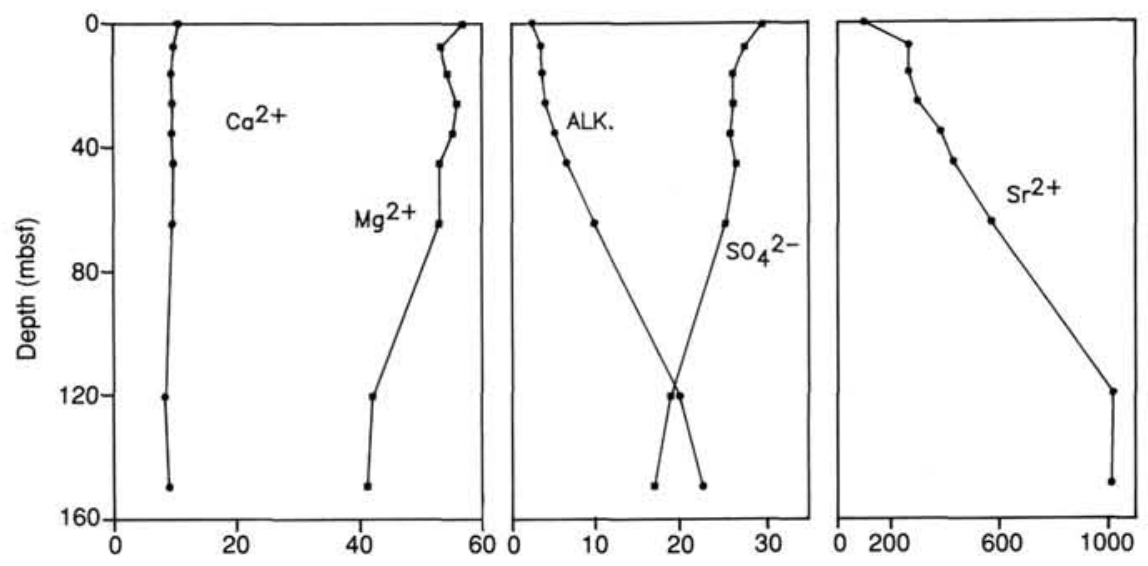

Figure 10. Interstitial pore-water chemistry, Hole 633A. See Figure 3 caption for units of value.

flux by the age of the sediment at depth, $z$ (Baker, 1981; Stout, 1985):

$$
\Sigma \mathrm{Sr}^{2+}=\left(\text { Flux }_{\mathrm{Sr}^{2+}}\right) \times(\text { Age }) .
$$

The minimum percentage of recrystallization is determined by dividing the total number of $\mathrm{Sr}^{2+}$ ions that have been removed from a given interval by the total amount of $\mathrm{Sr}^{2+}$ ions expected to be released to the pore fluids during recrystallization of the original sediment:

$$
\% \mathrm{R}=\frac{\Sigma \mathrm{Sr}^{2+}}{\text { Total } \mathrm{Sr}^{2+}}
$$

It is important to note that this gives only a minimum estimate of the percentage of sediment that has been recrystallized.

Intuitively, one would expect that the aragonite- and HMCdominated sediments cored during Leg 101 should rapidly recrystallize to a more stable mineralogy, but according to this method the computed rates of recrystallization are in fact lower than those in many pelagic carbonate deposits (Baker, 1981). 
$\mathrm{SrSO}_{4}$ IMP $\times 10^{-5}$

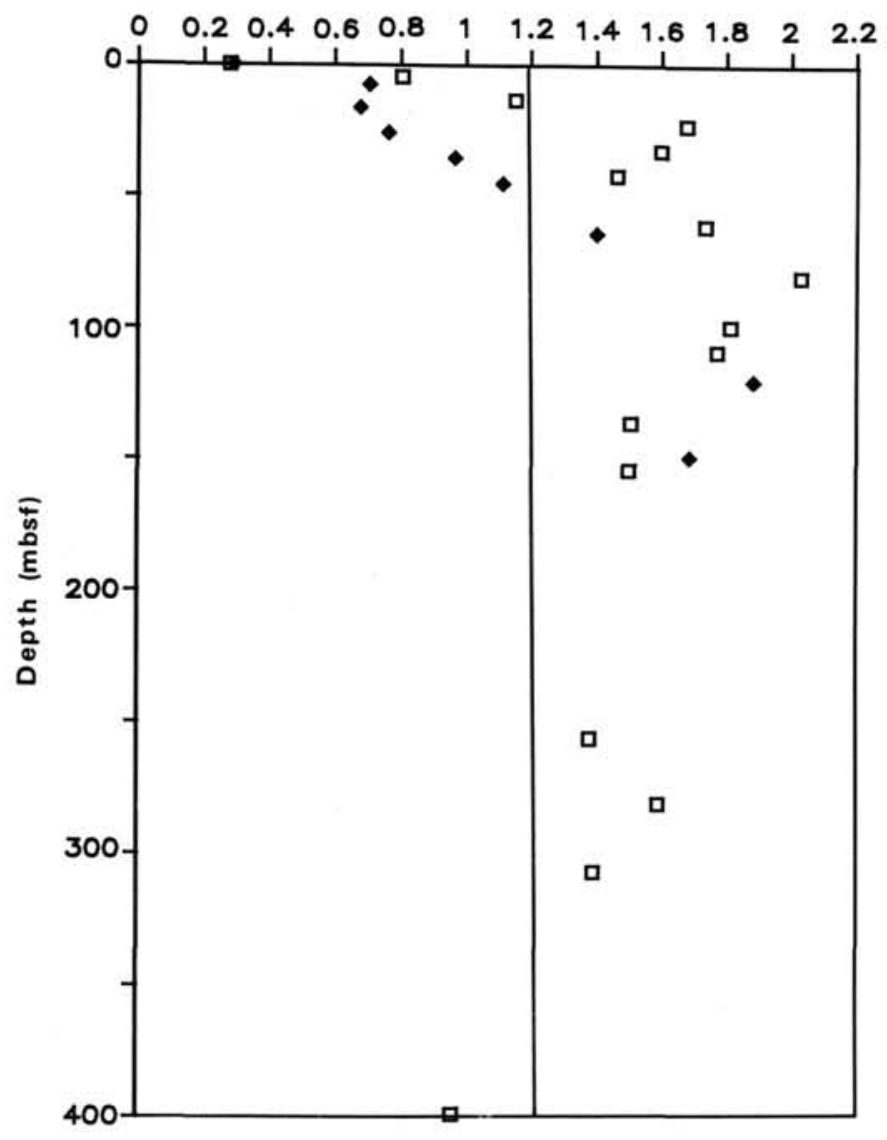

Figure 11. Ion molar product (IMP) of strontium sulfate from Holes 627B (squares) and 633A (diamonds). Vertical line through the diagram represents our best estimate of the $\mathrm{K}^{*} \mathrm{Sp}$ of celestite in marine waters (Culberson et al., 1978; MacDonald and North, 1974; Reardon and Armstrong, 1987). Points left of the vertical line represent samples undersaturated with respect to celestite, whereas points to the right are oversaturated with respect to celestite.

The low calculated recrystallization rates are mathematically a result of the high initial concentrations of strontium in periplatform sediments without proportionately steeper dissolved $\mathrm{Sr}^{2+}$ gradients. If the dissolved $\mathrm{Sr}^{2+}$ concentrations are limited by celestite precipitation, the above method gives erroneously low recrystallization rates. Therefore, although the derived rates can serve as minimum estimates, it is difficult to assess the true extent of diagenesis by this method because the amount of released strontium subsequently incorporated into celestite cannot be quantified.

A related but independent technique of quantifying diagenesis in carbonate deposits entails comparison of the measured $(\mathrm{Sr} / \mathrm{Ca})$ ratio of the sediment with the equilibrium $(\mathrm{Sr} / \mathrm{Ca}) \mathrm{ra}-$ tio, as predicted from pore-water chemistry (Baker, 1981; Baker et al., 1982; Delaney, 1983; Stout, 1985). The Sr/Ca ratios of the fine and coarse fractions for the samples from which pore waters were obtained are shown in Figures 12 through 18. The equilibrium $\mathrm{Sr} / \mathrm{Ca}$ ratios, as predicted from pore-water chemistry, are also shown (using a distribution coefficient of 0.035 ; Baker et al., 1982). Assuming that the pore-water $\mathrm{Sr}^{2+}$ and $\mathrm{Ca}^{2+}$ profiles have been relatively constant through time, the percentage of recrystallization can be estimated from the following equation:
Table 4. Bulk sediment mineralogy, channel sites.

\begin{tabular}{ccccc}
\hline $\begin{array}{c}\text { Depth } \\
(\mathrm{mbsf})\end{array}$ & $\begin{array}{c}\text { Calcite } \\
(\%)\end{array}$ & $\begin{array}{c}\text { Aragonite } \\
(\%)\end{array}$ & $\begin{array}{c}\text { Dolomite } \\
(\%)\end{array}$ & $\begin{array}{c}\text { Quartz } \\
(\%)\end{array}$ \\
\hline Hole 626C & & & & \\
54 & 100 & trace & 0 & 0 \\
121 & 100 & 0 & 0 & 0 \\
130 & 100 & 0 & trace & 0 \\
139 & 99 & 0 & 1 & 0 \\
169 & 75 & 25 & 0 & 0 \\
& & & & \\
Hole $626 \mathrm{D}$ & & & & \\
& & & 0 & 0 \\
189 & 87.0 & 13.0 & 0 & 0 \\
284 & 100.0 & 0 & 0.5 & 0 \\
313 & 99.5 & 0 & 0 & 0 \\
341 & 98.0 & 0 & 2.0 & 0 \\
370 & 95.2 & 2.6 & 2.2 & 0 \\
399 & 96.6 & 0 & 3.4 & 0 \\
418 & 84.4 & 0 & 15.6 & 0 \\
428 & 100.0 & 0 & 0 & 0 \\
447 & 100.0 & 0 & 0 & 0
\end{tabular}

Hole 634A

\begin{tabular}{rrrrr}
1.90 & 45 & 45 & 0 & 10 \\
2.90 & 86 & 0 & 12 & 3 \\
5.23 & 84 & 0 & 11 & 5 \\
146.90 & 97 & 0 & 0 & 3 \\
149.49 & 97 & 0 & 0 & 3 \\
164.70 & 96 & 0 & 2 & 2 \\
164.80 & 96 & 0 & 2 & 2 \\
165.90 & 97 & 0 & 0 & 3 \\
182.43 & 100 & 0 & 0 & 0 \\
229.90 & 100 & 0 & 0 & 0 \\
230.70 & 100 & 0 & 0 & 0 \\
258.60 & 100 & 0 & 0 & 0 \\
267.60 & 100 & 0 & 0 & 0 \\
354.70 & 100 & 0 & 0 & 0 \\
373.80 & 100 & 0 & 0 & 0 \\
441.10 & 95 & 0 & 3 & 2 \\
\hline
\end{tabular}

Table 5. Recrystallization (R) as calculated from $\mathbf{S r}^{2+}$ profiles.

\begin{tabular}{cccccr}
\hline Site & $\begin{array}{c}\text { Depth, } z \\
(\mathrm{mbsf})\end{array}$ & $\begin{array}{c}\text { Age at } z \\
(\mathrm{Ma})\end{array}$ & $\begin{array}{c}\text { Gradient } \\
(\mu \mathrm{mol} / \mathrm{L})\end{array}$ & $\begin{array}{c}\mathrm{\Sigma Sr}^{2+} \\
\left(\mathrm{mol} / \mathrm{cm}^{2}\right)\end{array}$ & $\% \mathrm{R}$ \\
\hline 627 & 60 & 5.0 & 10.0 & 0.055 & 12.0 \\
628 & 60 & 5.0 & 10.0 & 0.055 & 12.0 \\
630 & 140 & 7.2 & 3.7 & 0.030 & 2.8 \\
631 & 100 & 5.0 & 6.0 & 0.031 & 4.4 \\
632 & 100 & 5.0 & 6.0 & 0.031 & 4.4 \\
633 & 100 & 5.0 & 6.0 & 0.031 & 4.4 \\
\hline
\end{tabular}

Note: Sediment ages are from biostratigraphy of Watkins et al. (this volume).

$$
\% R=\frac{(\mathrm{Sr} / \mathrm{Ca})_{\mathrm{o}}-(\mathrm{Sr} / \mathrm{Ca})_{\mathrm{m}}}{(\mathrm{Sr} / \mathrm{Ca})_{\mathrm{o}}-(\mathrm{Sr} / \mathrm{Ca})_{\mathrm{e}}} \times 100,
$$

where

$(\mathrm{Sr} / \mathrm{Ca})_{0}=$ initial $(\mathrm{Sr} / \mathrm{Ca})$ ratio of sediment;

$(\mathrm{Sr} / \mathrm{Ca})_{\mathrm{m}}=$ measured $(\mathrm{Sr} / \mathrm{Ca})$ ratio of sediment;

$(\mathrm{Sr} / \mathrm{Ca})_{e}=$ predicted equilibrium $(\mathrm{Sr} / \mathrm{Ca})$ ratio.

Therefore, the depth of $100 \%$ recrystallization occurs where predicted equilibrium values begin to coincide with measured $\mathrm{Sr} / \mathrm{Ca}$ ratios; i.e., $(\mathrm{Sr} / \mathrm{Ca})_{\mathrm{m}}=(\mathrm{Sr} / \mathrm{Ca})_{\mathrm{e}}$. Depths at which this occurs in the LBB sites range from 40 to $80 \mathrm{~m}$ sub-bottom, except for the fine fraction from Hole $630 \mathrm{~A}$, which equilibrates at 
$\mathrm{Sr} / \mathrm{Ca}(\times 1000)$

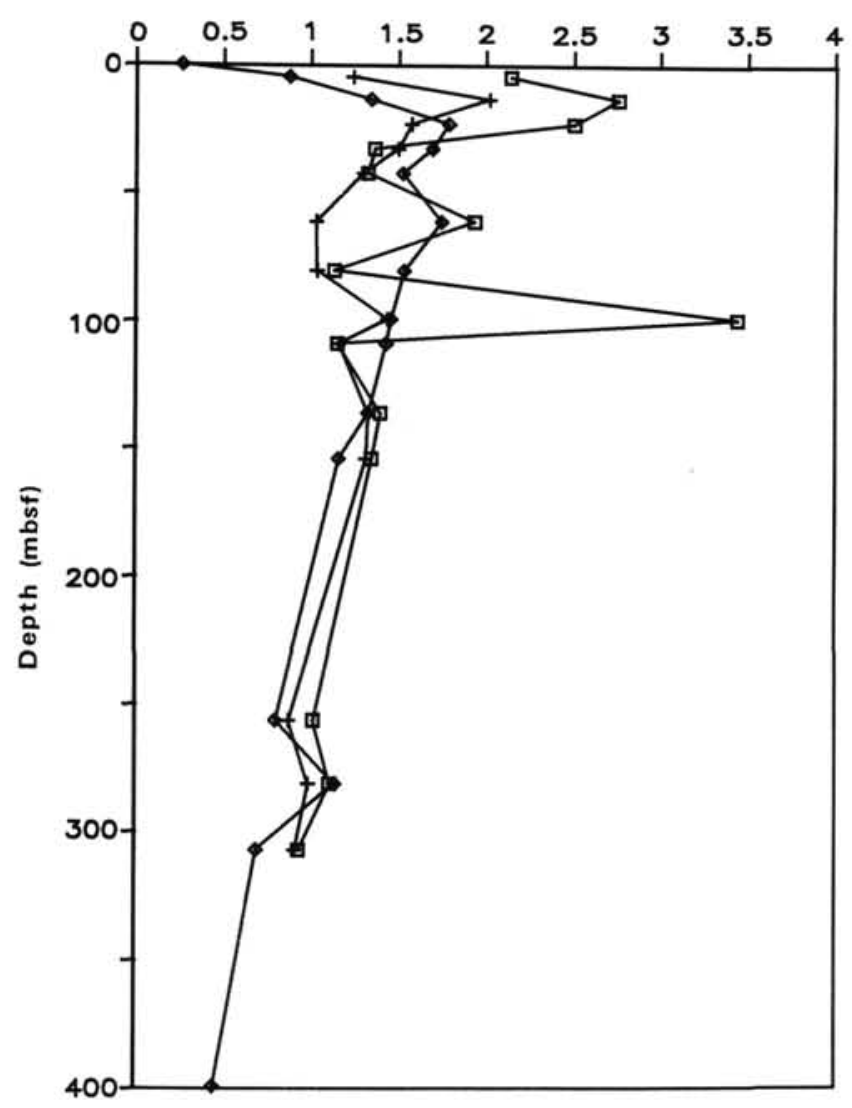

Figure 12. Sr/Ca ratio of coarse (pluses) and fine (squares) fractions of sediment from Hole 627B compared with the predicted $\mathrm{Sr} / \mathrm{Ca}$ ratio of calcite (diamonds) precipitated in equilibrium with ambient pore waters $\left(K_{\mathrm{Sr}}=0.035\right.$; Baker et al., 1982).

about $120 \mathrm{~m}$ sub-bottom. Interestingly, sediments from Hole $630 \mathrm{C}$ indicate that a significant amount of equilibration occurs within the upper $10 \mathrm{~m}$ of the cored section (Fig. 15). Overall, the derived depths are similar to, or slightly shallower than, those seen at some deep-sea pelagic sites (Baker, 1986). This would indicate, contrary to expectations, that recrystallization rates in these periplatform deposits are similar to those in pelagic carbonate deposits. However, as the area north of Little Bahama Bank did not experience true periplatform sedimentation throughout its entire history (Austin, Schlager, et al., 1986), the deposits present in the lower part of the cored section were initially pelagic and therefore should have been less susceptible to early recrystallization than periplatform sediments.

With the exception of Hole $631 \mathrm{~A}$, the $\mathrm{Sr} / \mathrm{Ca}$ ratios of the sediments in the Exuma Sound sites approach their expected equilibrium values at 100 to $120 \mathrm{~m}$ sub-bottom. The coincidence of $(\mathrm{Sr} / \mathrm{Ca})_{m}$ with $(\mathrm{Sr} / \mathrm{Ca})_{e}$ is somewhat surprising, as aragonitic needles still compose a significant part of the sediment in the zone of apparent equilibrium, suggesting that little recrystallization has taken place. For some reason, the strontium concentration of the aragonite component is lower than anticipated, which may in part explain why the recrystallization rates as calculated from the dissolved $\mathrm{Sr}^{2+}$ fluxes are lower than expected.

Although the profiles for Hole 631A show a downhole decrease in the measured $(\mathrm{Sr} / \mathrm{Ca})$ ratios within the upper $25 \mathrm{~m}$ of the section, they remain well above predicted equilibrium levels throughout the hole. The high $\mathrm{Sr} / \mathrm{Ca}$ ratio of the sediment sug-
$\mathrm{Sr} / \mathrm{Ca}(\times 1000)$

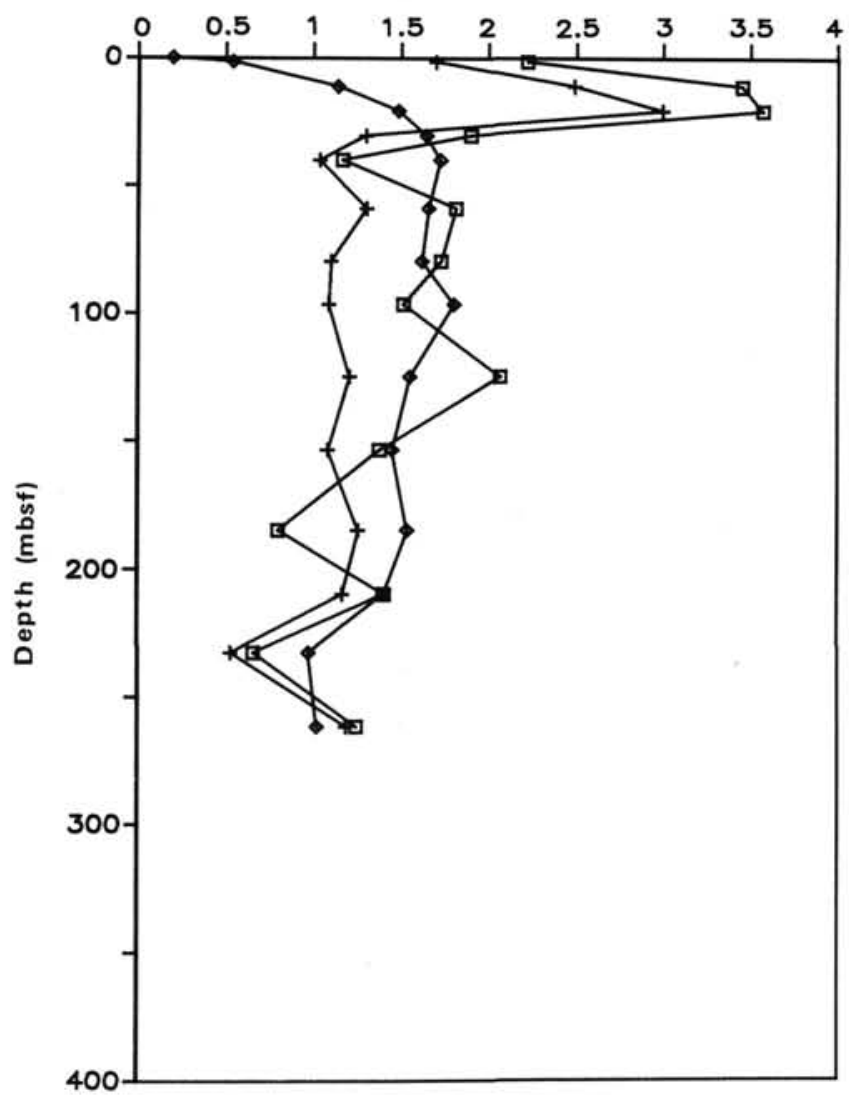

Figure 13. Sr/Ca ratio of coarse (pluses) and fine (squares) fractions of sediment from Hole $628 \mathrm{~A}$ compared with the predicted $\mathrm{Sr} / \mathrm{Ca}$ ratio of calcite (diamonds) precipitated in equilibrium with ambient pore waters $\left(\mathrm{K}_{\mathrm{Sr}}=0.035\right.$; Baker et al., 1982).

gests only partial recrystallization at this site. It is interesting to note that the measured profiles parallel the equilibrium profile, suggesting the presence of a constant diagenetic component. If a certain amount of the initial aragonite were to resist recrystallization, then the $\mathrm{Sr} / \mathrm{Ca}$ ratio of the sediment could always remain above that predicted for $100 \%$ diagenetic LMC. If we assume a $\mathrm{Sr}$ concentration for the measured aragonitic component $(7000 \mathrm{ppm})$ and deduct an appropriate amount of $\mathrm{Sr}$ from the $\mathrm{Sr}$ concentration of the bulk solids, we can then estimate the $\mathrm{Sr} / \mathrm{Ca}$ ratio of the LMC material. This exercise has been done for Hole 631A and is depicted graphically in Figure 19. Note the coincidence of the measured and predicted equilibrium levels beginning at only $70 \mathrm{~m}$ sub-bottom, indicating that the low-Mg calcitic component is in equilibrium with ambient pore fluids below this depth.

Alternatively, if a larger value for the distribution coefficient of $\mathrm{Sr}$ is employed (e.g., $\mathrm{K}_{\mathrm{Sr}}=0.1$ ), calculations indicate that $100 \%$ recrystallization has taken place by $40 \mathrm{~m}$ depth sub-bottom. While such a depth does not seem unreasonable, in view of the mineralogical data, this would require that the sediments be recrystallized to aragonite and not LMC, contrary to accepted theories of carbonate diagenesis in the deep marine environment. In addition, a small amount of residual celestite may have been contained within the solid fractions dissolved for Sr concentration analysis. Although none was detected using XRD, only a very small amount of $\mathrm{SrSO}_{4}$ is required to alter the results considerably. 
$\mathrm{Sr} / \mathrm{Ca}(\times 1000)$

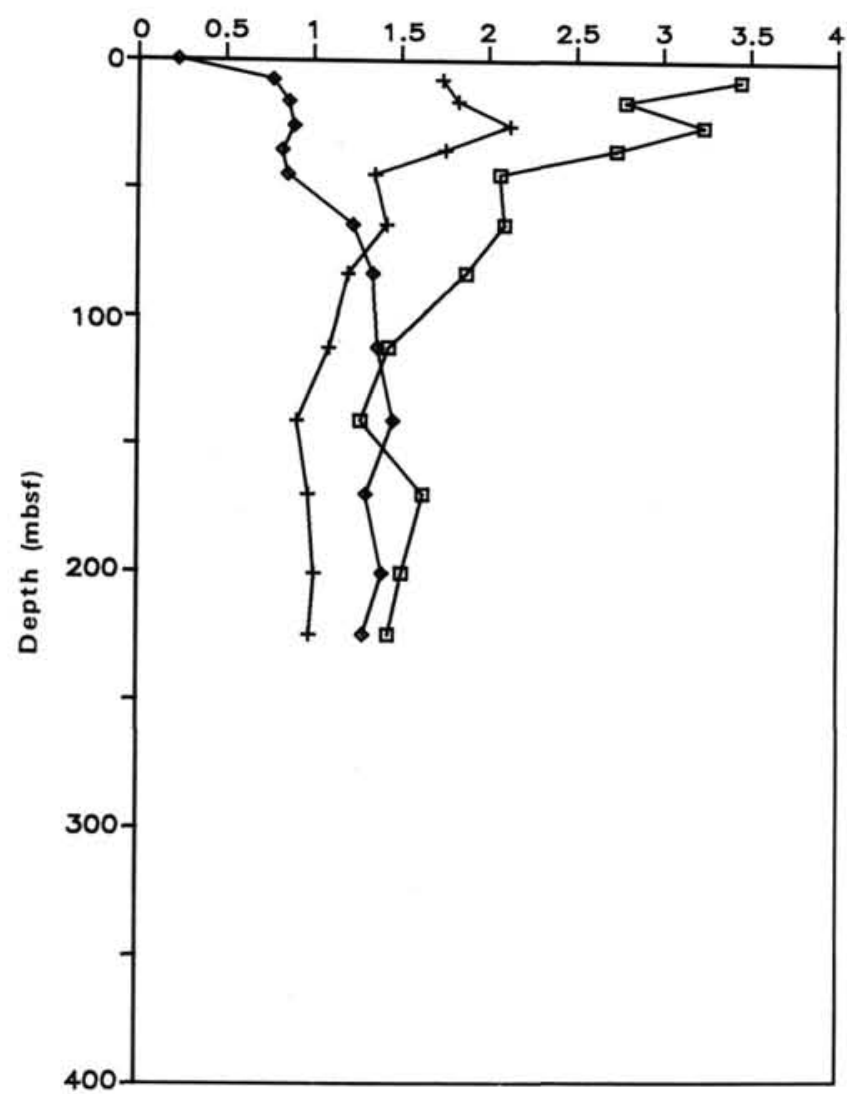

Figure 14. Sr/Ca ratio of coarse (pluses) and fine (squares) fractions of sediment from Hole 630A compared with the predicted $\mathrm{Sr} / \mathrm{Ca}$ ratio of calcite (diamonds) precipitated in equilibrium with ambient pore waters $\left(\mathrm{K}_{\mathrm{Sr}}=0.035\right.$; Baker et al., 1982).

\section{Celestite Precipitation}

The occurrence of celestite $\left(\mathrm{SrSO}_{4}\right)$ has been documented in deep-sea sediments cored during the DSDP (Schlanger et al., 1976; Kennett, von der Borch, et al., 1985; Baker and Bloomer, 1988). Celestite was detected in two samples (Samples 101-632A$7 \mathrm{H}-1,75 \mathrm{~cm}$, and 101-632B-3H, CC) collected during ODP Leg 101. The celestite observed at $150 \mathrm{~m}$ sub-bottom in Hole 632B (Sample 101-632B-3H, CC) was present as a fissure infill in carbonate material (Austin, Schlager, et al., 1986). The other documented occurrence of celestite from Leg 101 deposits (Sample $101-632 \mathrm{~A}-7 \mathrm{H}-1,75 \mathrm{~cm}$ ) was recognized only after leaching of the carbonate fraction.

Celestite occurrence is important to recrystallization studies because celestite precipitation can remove major quantities of dissolved $\mathrm{Sr}^{2+}$ from sediment pore waters. We believe that celestite precipitation is more common in Leg 101 deposits than the limited findings suggest. One obstacle to the detection of celestite is the nodular form which it commonly assumes in deep-sea deposits (Baker and Bloomer, 1988). Celestite of this type may be misidentified during sample description because of its visual similarity to carbonate minerals. Alternatively, if celestite were disseminated throughout the bulk sediment, it may also escape detection by standard XRD methods. A simple calculation reveals that only a small amount of celestite needs to be precipitated in order to account for the entire amount of $\mathrm{Sr}^{2+}$ released to the pore waters during the diagenesis of $\mathrm{Sr}$-rich biogenic arago-
$\mathrm{Sr} / \mathrm{Ca}(\times 1000)$

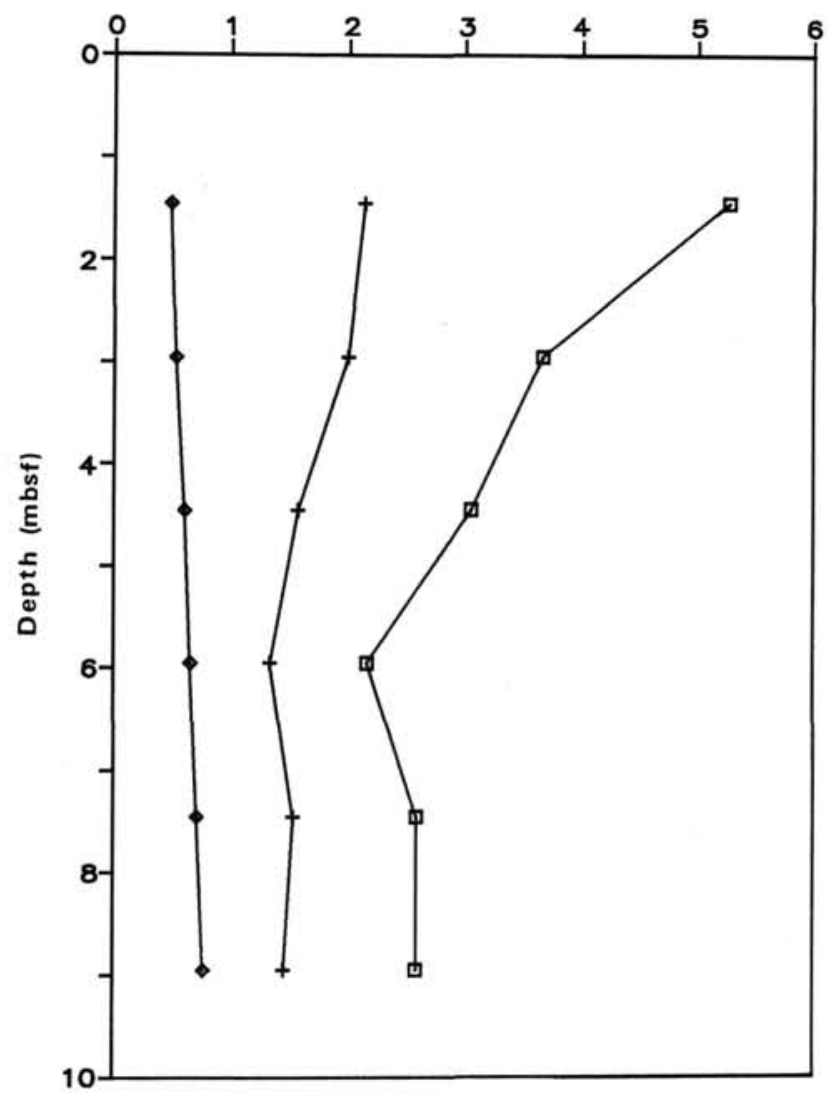

Figure 15. $\mathrm{Sr} / \mathrm{Ca}$ ratio of coarse (pluses) and fine (squares) fractions of sediment from Hole $630 \mathrm{C}$ compared with the predicted $\mathrm{Sr} / \mathrm{Ca}$ ratio of calcite (diamonds) precipitated in equilibrium with ambient pore waters $\left(\mathrm{K}_{\mathrm{Sr}}=0.035\right.$; Baker et al., 1982).

nite. If it is assumed that $1 \mathrm{~g}$ of aragonite with an initial $\mathrm{Sr}$ concentration equal to $10,000 \mathrm{ppm}$ dissolves and subsequently recrystallizes to $\mathrm{LMC}$ with a $\mathrm{Sr}$ concentration of $1000 \mathrm{ppm}$, there is a net loss of $0.009 \mathrm{~g}$ of $\mathrm{Sr}^{2+}$ to the pore fluids. This mass of strontium, if entirely precipitated as $\mathrm{SrSO}_{4}$, could form as much as $1.03 \times 10^{-4} \mathrm{~mol}$ of celestite. This is equivalent to only 0.019 $\mathrm{g}$ of $\mathrm{SrSO}_{4}$. Celestite would thus compose, at most, less than $2 \%$ of the bulk sediment. It is quite possible that such a minute amount of celestite would not be detected in the X-ray analyses of bulk-sediment samples.

An equal quantity of $\mathrm{Sr}^{2+}$ and $\mathrm{SO}_{4}^{2-}$ is required for the formation of celestite. Consequently, if the entire amount of $\mathrm{Sr}^{2+}$ released by the recrystallization of our hypothetical $1 \mathrm{~g}$ of aragonitic sediment were to be precipitated as celestite, $1.03 \times 10^{-4}$ mol of $\mathrm{SO}_{4}^{2-}$ would be required. The $0.36 \mathrm{~cm}^{3}$ of pore fluid associated with this $1 \mathrm{~g}$ of celestite would typically contain only $1.008 \times 10^{-5} \mathrm{~mol}$ of $\mathrm{SO}_{4}^{2+}$, an order of magnitude less than that required. Although this is a maximum estimate for the amount of $\mathrm{SO}_{4}^{2-}$ needed, this calculation shows that the precipitation of celestite might have a profound effect on pore-waterdissolved $\mathrm{SO}_{4}^{2-}$ profiles as well as dissolved $\mathrm{Sr}^{2+}$ profiles.

\section{Dolomitization}

As in previous studies of periplatform sediments (Droxler, 1984; Mullins et al., 1984; Eaton and Boardman, 1985; and others), dolomite was detected in many of the samples collected 
$\mathrm{Sr} / \mathrm{Ca}(\times 1000)$

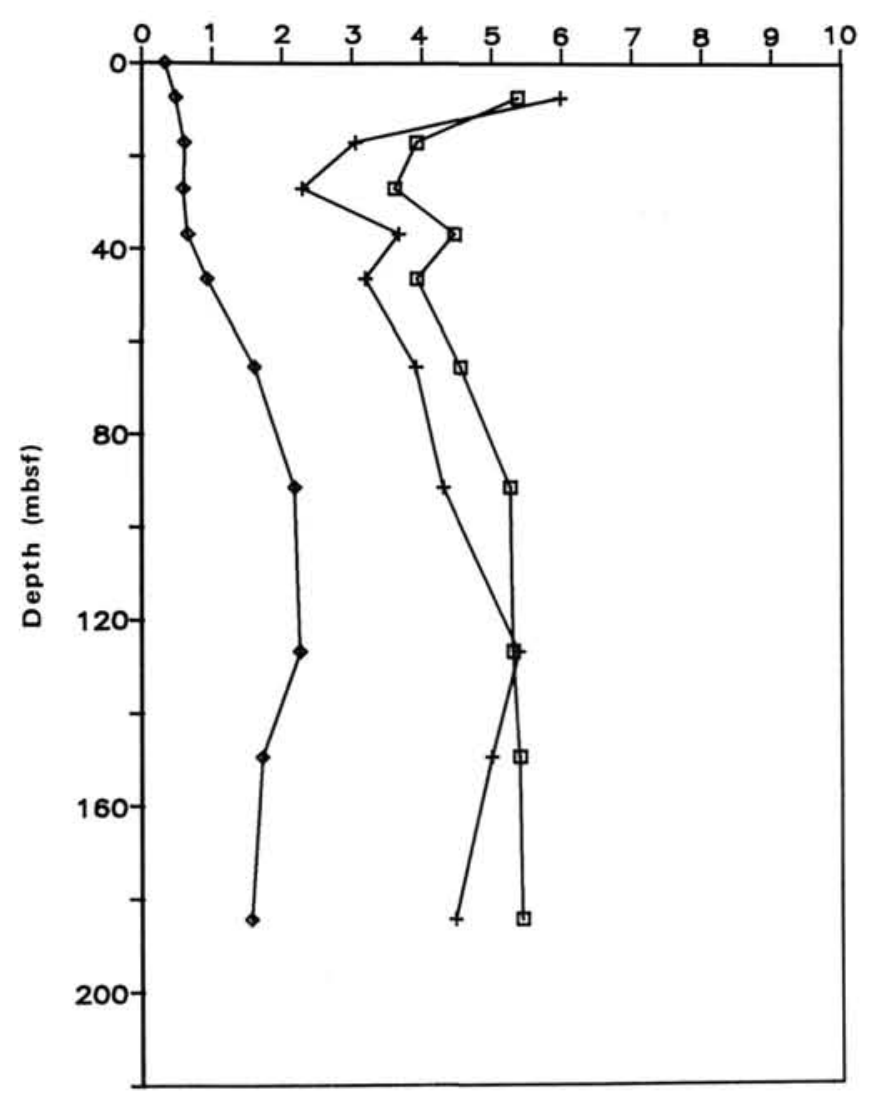

Figure 16. Sr/Ca ratio of coarse (pluses) and fine (squares) fractions of sediment from Hole 631A compared with the predicted $\mathrm{Sr} / \mathrm{Ca}$ ratio of calcite (diamonds) precipitated in equilibrium with ambient pore waters $\left(\mathrm{K}_{\mathrm{Sr}}=0.035\right.$; Baker et al., 1982).

during Leg 101. Typically, dolomite composes up to $10 \mathrm{wt} \%$ of the sediment, but concentrations reach as high as $40 \mathrm{wt} \%$ (e.g., at $167 \mathrm{~m}$ sub-bottom in Hole 633A). The origin of the dolomite, however, remains a matter of speculation, as both authigenic (Mullins et al., 1984) and detrital (Eaton and Boardman, 1985) sources have been suggested. Both types of dolomite are likely to be present in these sediments, but for purposes of this discussion, we consider only the conditions necessary for an authigenic origin.

Authigenic dolomitization that proceeds according to one of the following equations requires an adequate source of $\mathrm{Mg}^{2+}$, $\mathrm{CO}_{3}^{2-}$, and $\mathrm{HCO}_{3}^{-}$ions, as well as a mechanism for their transport to the zone of dolomitization:

$$
\begin{gathered}
2 \mathrm{CaCO}_{3}+\mathrm{Mg}^{2+} \rightleftharpoons \mathrm{CaMg}\left(\mathrm{CO}_{3}\right)_{2}+\mathrm{Ca}^{2+} ; \\
\mathrm{CaCO}_{3}+\mathrm{Mg}^{2+}+\mathrm{CO}_{3}^{2+} \rightleftharpoons \mathrm{CaMg}_{\left(C_{3}\right)_{2} ;} \\
\mathrm{CaCO}_{3}+\mathrm{Mg}^{2+}+\mathrm{HCO}_{3}^{-} \rightleftharpoons \mathrm{CaMg}_{\left(\mathrm{CO}_{3}\right)_{2}+\mathrm{H}^{+}} .
\end{gathered}
$$

In regard to the source of $\mathrm{Mg}^{2+}$, there are only two viable possibilities, either the local dissolution of $\mathrm{Mg}$-rich minerals or seawater itself.

Dissolution of local HMC is the most plausible mineral source of $\mathrm{Mg}^{2+}$ ions. Upon dissolution of $\mathrm{HMC}, \mathrm{Mg}^{2+}$ is released to pore waters and becomes available for dolomite formation. For

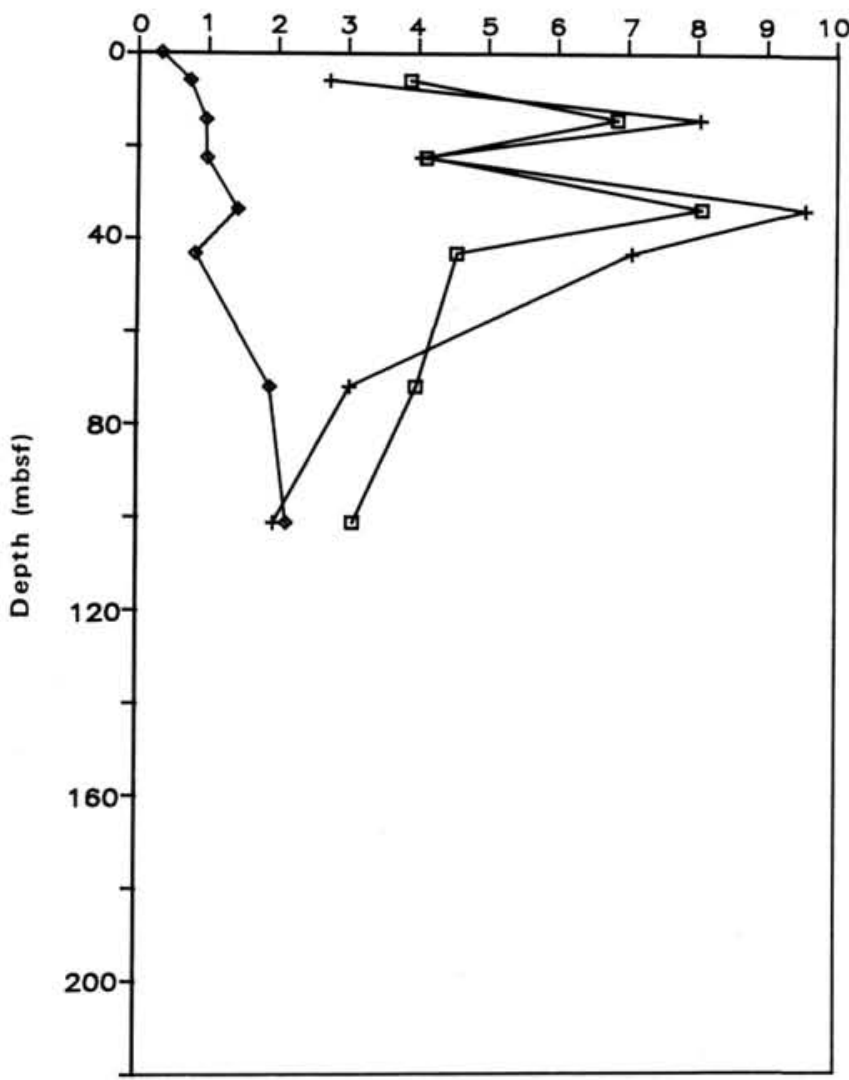

Figure 17. $\mathrm{Sr} / \mathrm{Ca}$ ratio of coarse (pluses) and fine (squares) fractions of sediment from Hole $632 \mathrm{~A}$ compared with the predicted $\mathrm{Sr} / \mathrm{Ca}$ ratio of calcite (diamonds) precipitated in equilibrium with ambient pore waters $\left(\mathrm{K}_{\mathrm{Sr}}=0.035\right.$; Baker et al., 1982).

example, approximately $8 \mathrm{wt} \%$ dolomite could be formed from a sediment containing $20 \%$ HMC with a composition of $5 \mathrm{wt} \%$ $\mathrm{Mg}\left(20 \mathrm{~mol}_{\%} \mathrm{MgCO}_{3}\right)$, if all the $\mathrm{Mg}$ released from the HMC were reincorporated into dolomite. With only a few exceptions, HMC disappears (i.e., dissolves or was not initially present) in the sediments in the upper 1 to $10 \mathrm{~m}$. Therefore, if the $\mathrm{Mg}^{2+}$ ions are derived from this source, the dolomite must have formed near the sediment/water interface.

An alternative source of $\mathrm{Mg}^{2+}$ might be that provided by the interstitial pore waters. Because of the small amount of $\mathbf{M g}^{2+}$ available in any one volume of pore fluid $(1 \mathrm{~g}$ of sediment with $50 \%$ porosity contains only $1.7 \times 10^{-5} \mathrm{~mol} \mathrm{Mg}^{2+}$ ), the cations must move to the site of dolomitization by either diffusion or convection.

Let us first consider the contribution of $\mathrm{Mg}^{2+}$ ions as a result of molecular diffusion. For illustrative purposes, we will consider a $\left(1-\mathrm{cm}^{2}\right)$ column of sediment having the chemistry and mineralogy of Hole $633 \mathrm{~A}$, as this hole shows the highest overall dolomite concentrations. The present-day flux of $\mathrm{Mg}^{2+}$ to a given depth, $z$, can be calculated from Fick's Law (Equation 1). Assuming $\mathrm{D}_{\mathrm{b}}=3.5 \times 10^{-6} \mathrm{~cm}^{2} / \mathrm{s}$ (Compton and Siever, 1986), solution of this equation gives a present-day $\mathrm{Mg}^{2+}$ flux of $3.75 \times 10^{-15} \mathrm{~mol} / \mathrm{cm}^{2} / \mathrm{s}\left(0.12 \mathrm{~mol} / \mathrm{cm}^{2} / \mathrm{Ma}\right)$. If we presume that dolomitization is now occurring in a $10-\mathrm{m}$ zone between 150 and $160 \mathrm{~m}$ sub-bottom, operation of this flux over $1 \mathrm{~m} . \mathrm{y}$. would contribute enough $\mathrm{Mg}^{2+}$ to this interval to form $21.81 \mathrm{~g}$ 
$\mathrm{Sr} / \mathrm{Ca}(\times 1000)$

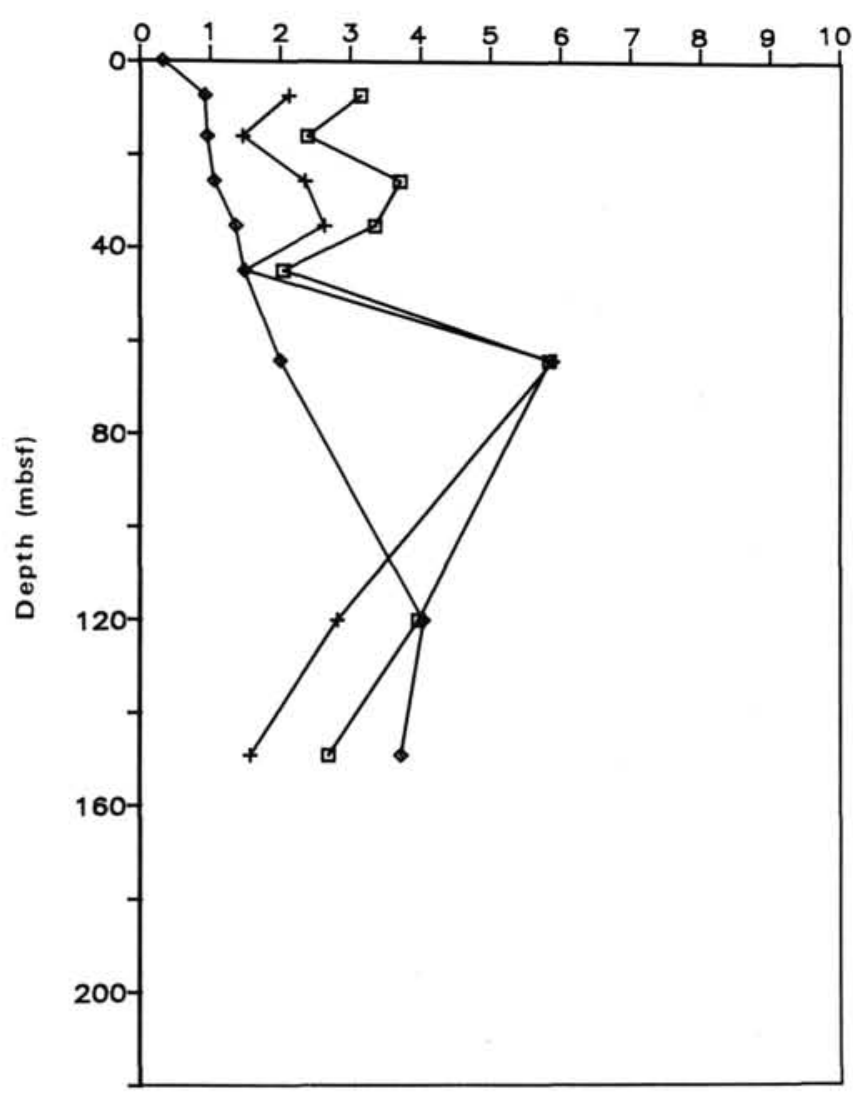

Figure 18. Sr/Ca ratio of coarse (pluses) and fine (squares) fractions of sediment from Hole 633A compared with the predicted $\mathrm{Sr} / \mathrm{Ca}$ ratio of calcite (diamonds) precipitated in equilibrium with ambient pore waters $\left(\mathrm{K}_{\mathrm{Sr}}=0.035\right.$; Baker et al., 1982).

of dolomite. In a sediment with a porosity of $50 \%$, this amount of dolomite represents only $1.6 \mathrm{wt} \%$ of the sediment, a small amount in comparison to the average of $10 \mathrm{wt} \%$ dolomite present throughout most of Hole 633A. Evidently, the present-day $\mathrm{Mg}^{2+}$ flux is insufficient to account for the dolomite concentrations in Hole 633A. It is possible that paleogradients of $\mathrm{Mg}^{2+}$ were steeper than the modern gradient. This would accelerate $\mathrm{Mg}^{2+}$ flux rates and allow for proportionately greater rates of dolomite formation.

An alternative mechanism for the transport of $\mathrm{Mg}^{2+}$ ions is the convection of fluid through the sediments, as suggested by Simms (1984). This has previously been invoked as a mechanism for providing $\mathrm{Mg}^{2+}$ ions for dolomite formation in piston cores taken north of Little Bahama Bank (Mullins et al., 1984). However, if fluid convection was important in these deposits, it would be impossible to maintain the measured chemical gradients. Let us first consider downward convection of seawater at a darcy velocity of $1 \mathrm{~m} / \mathrm{yr}$, as suggested by Simms (1984). Such a flux would necessitate an unreasonably high rate of recrystallization $(2.56 \%$ per $1000 \mathrm{yr})$ in order to maintain a $\mathrm{Sr}^{2+}$ gradient of 6 $\mu \mathrm{mol} / \mathrm{m}$, typical of the Exuma Sound sites. At such a rate, by 39,000 yr post-deposition, there would no longer be any $\mathrm{Sr}^{2+}$ release associated with sediment recrystallization, thus rendering the persistence of any $\mathrm{Sr}^{2+}$ gradient below this depth unlikely. In addition, aragonite would not be present in sediments older than late Pleistocene, contrary to the mineralogical data from

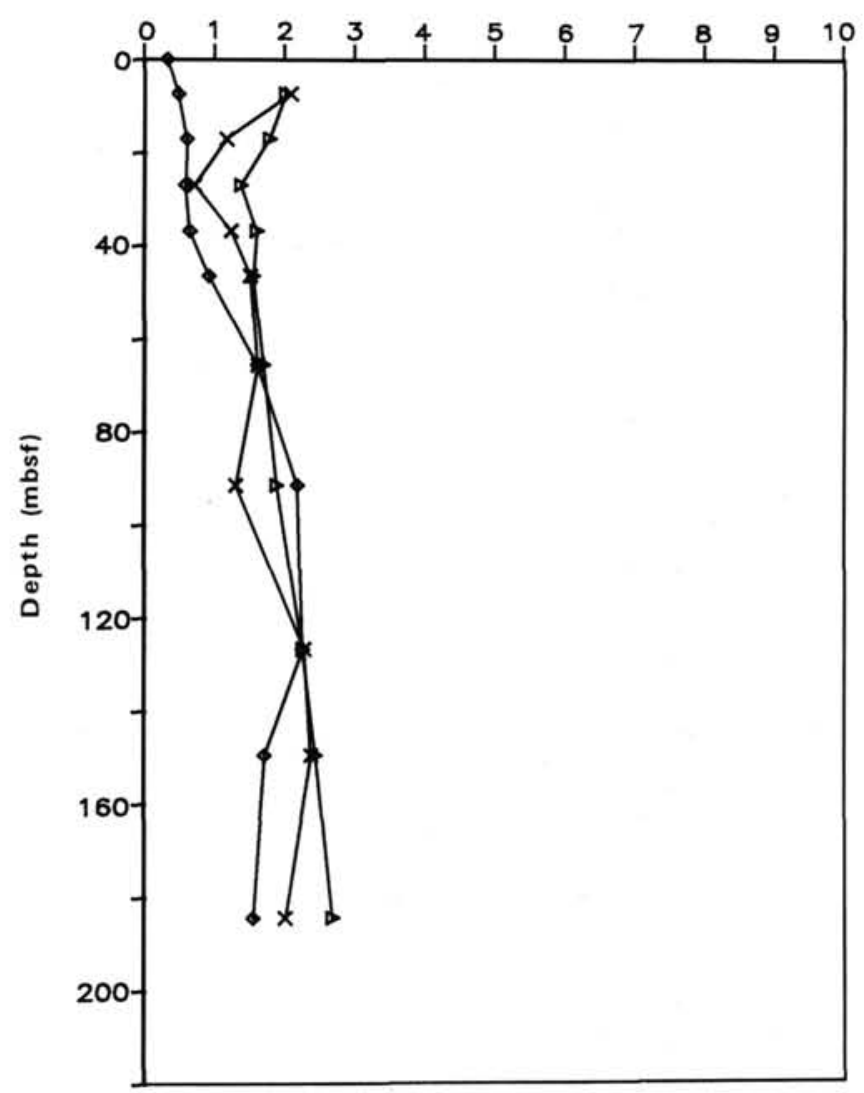

Figure 19. Estimated $\mathrm{Sr} / \mathrm{Ca}$ ratios of coarse $(X \mathrm{~s})$ and fine (triangles) LMC fraction of sediment from Hole 631A compared with the predicted $\mathrm{Sr} / \mathrm{Ca}$ ratio of calcite (diamonds) precipitated in equilibrium with ambient pore waters $\left(\mathrm{K}_{\mathrm{Sr}}=0.035\right.$; Baker et al., 1982). The estimated $\mathrm{LMC} \mathrm{Sr} / \mathrm{Ca}$ ratios reflect subtraction of the calculated content of $\mathrm{Sr}$ in associated aragonite from the bulk-sediment total $\mathrm{Sr}$ concentration. A concentration of $7000 \mathrm{ppm}$ was assumed for the aragonitic fraction.

these deposits (Tables 1, 3, and 6). Thus, downward fluid convection would require that sediments be recrystallized at excessively high rates in order to maintain the observed geochemical gradients.

Upward convection from the underlying deposits is also disproven by the existing chemical gradients. If $\mathrm{Mg}^{2+}$ ions were in fact derived from the pore fluids as they moved upward through the sediments, then we would not expect to see a negative dissolved $\mathrm{Mg}^{2+}$ gradient such as exists in Hole 633A (downward molecular diffusion from overlying seawater is extremely slow in comparison to the required rates of upward fluid convection). We therefore believe that no significant amount of vertical fluid convection is now occurring within these deposits.

In conclusion, our calculations suggest that while authigenic dolomite formation may be occurring in the subsurface of these deposits, diffusion of $\mathrm{Mg}^{2+}$ from overlying seawater according to the present gradient does not supply enough $\mathrm{Mg}^{2+}$ to account for the observed dolomite concentrations. It is possible that $\mathrm{Mg}^{2+}$ gradients were somewhat steeper in the past when the zone of dolomitization was nearer the surface, or that a large proportion of dolomite formation occurred concomitant with the dissolution of HMC. This latter source is sufficient to account for the highest observed dolomite concentrations only if the sediment was initially composed of over $90 \mathrm{wt} \% \mathrm{HMC}$. The present 
Table 6. Mineralogy of coarse $(>63 \mu \mathrm{m})$ and fine $(<63 \mu \mathrm{m})$ fractions, Leg 101.

\begin{tabular}{|c|c|c|c|c|c|c|c|c|c|c|c|c|}
\hline $\begin{array}{l}\text { Core, section, and } \\
\text { sample interval }(\mathrm{cm})\end{array}$ & $\begin{array}{c}\text { Dolomite } \\
\text { fines } \\
\text { (wt \%) }\end{array}$ & $\begin{array}{c}\text { Calcite } \\
\text { fines } \\
\text { (wt \%) }\end{array}$ & $\begin{array}{c}\text { Aragonite } \\
\text { fines } \\
\text { (wt } \%)\end{array}$ & $\begin{array}{c}\text { Quartz } \\
\text { fines } \\
\text { (wt \%) }\end{array}$ & $\begin{array}{c}\text { Dolomite } \\
\text { coarse } \\
\text { (wt \%) }\end{array}$ & $\begin{array}{l}\text { Calcite } \\
\text { coarse } \\
(w t \%)\end{array}$ & $\begin{array}{l}\text { Aragonite } \\
\text { coarse } \\
\text { (wt } \%)\end{array}$ & $\begin{array}{l}\text { Quartz } \\
\text { coarse } \\
\text { (wt \%) }\end{array}$ & $\begin{array}{c}\text { Fines } \\
(\%)\end{array}$ & $\begin{array}{c}\text { Coarse } \\
(\%)\end{array}$ & $\begin{array}{c}\mathrm{Sr} \\
\text { fines } \\
(\mathrm{ppm})\end{array}$ & $\begin{array}{c}\mathrm{Sr} \\
\text { coarse } \\
(\mathrm{ppm})\end{array}$ \\
\hline \multicolumn{13}{|l|}{ Hole $626 \mathrm{C}$} \\
\hline $3 X-3,140-150$ & 3.00 & 92.5 & 3.8 & 0.70 & 4.3 & 89.7 & 4.70 & 1.3 & 22.62 & 77.38 & 2320 & - \\
\hline $14 \mathrm{H}-5,140-150$ & 5.30 & 89.1 & 3.5 & 2.10 & 4.7 & 95.3 & 0 & 0 & 38.40 & 61.60 & 1737 & 889 \\
\hline $15 \mathrm{H}-5,140-150$ & 5.80 & 88.5 & 3.3 & 2.40 & 6.5 & 93.5 & 0 & 0 & 38.01 & 61.99 & 1737 & 846 \\
\hline $18 \mathrm{H}-5,140-150$ & 4.00 & 89.7 & 4.7 & 1.60 & 4.5 & 94.7 & 0 & 0.8 & 42.15 & 57.85 & 2298 & 971 \\
\hline \multicolumn{13}{|l|}{ Hole 627A } \\
\hline $1 \mathrm{H}-3,140-150$ & 4.70 & 66.9 & 24.1 & 4.20 & 3.0 & 96.3 & 0.70 & 0 & - & - & - & - \\
\hline \multicolumn{13}{|l|}{ Hole 627B } \\
\hline $1 \mathrm{H}-3,140-150$ & 13.30 & 64.6 & 0 & 22.10 & 5.7 & 89.6 & 0.30 & 4.4 & 97.82 & 2.18 & - & 1085 \\
\hline $2 \mathrm{H}-5,140-150$ & 4.00 & 62.3 & 30.8 & 2.60 & 3.3 & 94.2 & 2.30 & 0.3 & 81.45 & 18.55 & 2414 & 1770 \\
\hline $3 \mathrm{H}-5,140-150$ & 5.70 & 72.5 & 19.6 & 2.10 & 3.0 & 96.3 & 0 & 0.7 & 78.26 & 21.74 & 2194 & 1378 \\
\hline $4 \mathrm{H}-5,140-150$ & 6.00 & 72.6 & 18.3 & 3.10 & 3.5 & 96.5 & 0 & 0 & 62.49 & 37.51 & 1196 & 1311 \\
\hline $5 \mathrm{H}-5,140-150$ & 6.50 & 90.3 & 0 & 3.20 & 12.1 & 87.9 & 0 & 0 & 69.55 & 30.45 & 1160 & 9455 \\
\hline $7 \mathrm{H}-5,140-150$ & 3.00 & 75.2 & 21.6 & 0.20 & 2.5 & 96.7 & 0 & 0.8 & 41.95 & 58.05 & 1697 & 906 \\
\hline $9 \mathrm{H}-5,140-150$ & 4.00 & 93.7 & 0 & 2.30 & 4.5 & 95.5 & 0 & 0 & 53.62 & 46.38 & 997 & 909 \\
\hline $11 \mathrm{H}-5,140-150$ & 2.60 & 59.0 & 37.3 & 1.10 & 2.6 & 97.4 & 0 & 0 & 84.10 & 15.90 & 3017 & 1263 \\
\hline $12 \mathrm{H}-5,140-150$ & 4.20 & 93.0 & 0 & 2.80 & 4.0 & 94.7 & 0 & 1.4 & 79.11 & 20.89 & 1012 & 1020 \\
\hline $15 \mathrm{H}-4,140-150$ & 4.60 & 91.1 & 0.3 & 4.05 & 4.8 & 95.2 & 0 & 0 & 96.98 & 3.02 & 1226 & 1165 \\
\hline $17 X-3,140-150$ & 6.00 & 92.3 & 0 & 1.70 & 6.1 & 93.9 & 0 & 0 & 71.73 & 28.27 & 1184 & 1156 \\
\hline $21 X-3,140-150$ & 2.70 & 86.6 & 9.0 & 1.80 & 4.7 & 92.6 & 1.80 & 0.9 & 45.02 & 54.98 & 1309 & 1353 \\
\hline $27 X-5,140-150$ & 4.50 & 94.0 & 0 & 1.50 & 1.5 & 98.5 & 0 & 0 & 99.60 & 0.40 & 896 & 774 \\
\hline $30 X-3,140-150$ & 4.20 & 95.8 & 0 & 0 & 3.8 & 96.2 & 0 & 0 & 99.63 & 0.37 & 979 & 872 \\
\hline $33 \mathrm{X}-1,140-150$ & 3.40 & 95.2 & 0 & 1.50 & 2.6 & 97.4 & 0 & 0 & 98.07 & 1.93 & 823 & 806 \\
\hline \multicolumn{13}{|l|}{ Hole $628 \mathrm{~A}$} \\
\hline $1 \mathrm{H}-1,140-150$ & 4.90 & 67.3 & 25.8 & 2.00 & 1.7 & 80 & 18.30 & 0 & 66.53 & 33.47 & 1947 & 1489 \\
\hline $2 \mathrm{H}-5,140-150$ & 5.00 & 53.4 & 40.5 & 1.04 & 4.4 & 67.0 & 28.60 & 0 & 58.78 & 41.22 & 3029 & 2185 \\
\hline $3 \mathrm{H}-5,140-150$ & 2.20 & 53.8 & 43.1 & 0.90 & 5.1 & 63.0 & 30.50 & 1.4 & 43.13 & 56.87 & 3130 & 2626 \\
\hline $4 \mathrm{H}-5,140-150$ & 4.70 & 78.8 & 14.9 & 1.50 & 4.1 & 95.6 & 0.37 & 0 & 61.78 & 38.22 & 1666 & 1142 \\
\hline $5 \mathrm{H}-5,140-150$ & 13.80 & 81.7 & 4.5 & 0 & 7.9 & 92.0 & 0.10 & 0 & 34.98 & 65.02 & 1026 & 911 \\
\hline $7 \mathrm{H}-5,140-150$ & 4.60 & 92.4 & 1.7 & 1.30 & 5.9 & 92.2 & 0 & 1.9 & 84.82 & 15.18 & 1592 & 1148 \\
\hline $9 \mathrm{H}-4,140-150$ & 6.70 & 90.1 & 2.3 & 0.80 & 5.3 & 94.4 & 0.30 & 0 & 94.14 & 5.86 & 1519 & 968 \\
\hline $11 \mathrm{H}-5,140-150$ & 5.30 & 88.3 & 6.3 & 0 & 2.8 & 97.2 & 0 & 0 & 65.72 & 34.28 & 1331 & 958 \\
\hline $14 \mathrm{H}-5,140-150$ & 1.85 & 79.9 & 17.7 & 0.50 & 3.7 & 96.3 & 0 & 0 & 89.11 & 10.89 & 1817 & 1066 \\
\hline $17 \mathrm{H}-5,140-150$ & 3.20 & 92.9 & 0 & 4.00 & 2.5 & 95.0 & 1.00 & 1.5 & 95.96 & 4.04 & 1215 & 955 \\
\hline $21 \mathrm{H}-2,0-10$ & 4.11 & 93.0 & 0 & 2.90 & 4.0 & 94.0 & 0 & 2.0 & 96.73 & 3.27 & 708 & 1109 \\
\hline $23 \mathrm{H}-5,140-150$ & 2.40 & 97.6 & 0 & 0 & 3.7 & 96.3 & 0 & 0 & 91.41 & 8.59 & 1238 & 1029 \\
\hline $26 \mathrm{X}-1,140-150$ & 2.00 & 45.0 & 49.3 & 3.70 & 2.7 & 94.9 & 0 & 2.4 & 65.47 & 34.53 & 585 & 471 \\
\hline $29 \mathrm{X}-1,140-150$ & 3.43 & 94.2 & 0 & 2.30 & 5.5 & 94.5 & 0 & 0 & 99.44 & 0.56 & 1099 & 1049 \\
\hline \multicolumn{13}{|l|}{ Hole $630 \mathrm{~A}$} \\
\hline $1 \mathrm{H}-5,140-150$ & 1.10 & 61.0 & 37.2 & 0.60 & 3.3 & 75.0 & 19.90 & 0.8 & 91.60 & 8.40 & 3016 & 1521 \\
\hline $2 \mathrm{H}-5,140-150$ & 4.90 & 64.6 & 29.4 & 1.10 & 1.8 & 83.0 & 14.50 & 0.7 & 91.62 & 8.38 & 2437 & 1600 \\
\hline $3 \mathrm{H}-5,140-150$ & 4.50 & 62.0 & 33.5 & 0 & 1.3 & 76.0 & 22.00 & 0.7 & 905 & 9.95 & 2831 & 1860 \\
\hline $4 \mathrm{H}-5,140-150$ & 5.60 & 62.9 & 30.6 & 0.90 & 2.3 & 91.5 & 5.00 & 1.2 & 85.92 & 14.08 & 2392 & 1536 \\
\hline $5 \mathrm{H}-5,140-150$ & 6.40 & 74.3 & 18.5 & 0.70 & 5.3 & 94.7 & 0 & 0 & 77.37 & 22.63 & 1812 & 1186 \\
\hline $7 \mathrm{H}-5,140-150$ & 2.20 & 78.4 & 18.1 & 1.30 & 3.3 & 96.0 & 0 & 0.7 & 85.94 & 14.06 & 1834 & 1244 \\
\hline $9 \mathrm{H}-5,140-150$ & 4.20 & 78.0 & 16.0 & 1.80 & 2.0 & 97.6 & 0 & 0.4 & 86.94 & 13.06 & 1644 & 1054 \\
\hline $12 \mathrm{H}-5,140-150$ & 6.90 & 90.1 & 0 & 3.00 & 7.1 & 92.9 & 0 & 0 & 84.25 & 15.75 & 1255 & 956 \\
\hline $15 \mathrm{H}-5,140-150$ & 6.60 & 87.6 & 4.9 & 0.90 & 10.3 & 88.9 & 0 & 0.8 & 76.24 & 23.76 & 1117 & 800 \\
\hline $18 \mathrm{H}-5,140-150$ & 7.40 & 80.8 & 10 & 1.80 & 8.8 & 87.0 & 3.00 & 1.2 & 51.84 & 48.16 & 1427 & 857 \\
\hline $21 X-5,140-150$ & 6.20 & 82.0 & 10 & 0.80 & 4.2 & 95.8 & 0 & 0 & 71.57 & 28.43 & 1324 & 888 \\
\hline $24 \mathrm{X}-2,140-150$ & 4.60 & 85.0 & 9.9 & 0.50 & 7.2 & 92.8 & 0 & 0 & 70.55 & 29.45 & 1256 & 864 \\
\hline
\end{tabular}

concentration of HMC in the surface sediments of these deposits is actually less than $15 \mathrm{wt} \%$ (Austin, Schlager, et al., 1986). A further consideration is that some amount of the dolomite is probably detrital in origin.

\section{$\mathrm{Ca}^{2+}$ Gradients as Stratigraphic Tools}

Accepting the premise that $\mathrm{Ca}^{2+}$ gradients observed at several sites drilled in the Bahamas are a result of diffusion from a common underlying source (the evaporitic unit encountered in the Albian section of Hole 627B), the depth to this unit at other, shallower holes that did not actually penetrate this unit can be approximated. By extrapolation of the $\mathrm{Ca}^{2+}$ gradient at Hole
$627 \mathrm{~B}$, pore waters within the evaporitic formation are estimated to have $\mathrm{Ca}^{2+}$ concentrations of $35 \mathrm{mmol} / \mathrm{L}$. If we then extrapolate the dissolved $\mathrm{Ca}^{2+}$ gradients at Hole $628 \mathrm{~A}(0.031 \mathrm{mmol} / \mathrm{m})$ and at Hole $630 \mathrm{~A}(0.026 \mathrm{mmol} / \mathrm{m})$, we find that a concentration of $35 \mathrm{mmol} / \mathrm{L}$ is reached at 800 and $960 \mathrm{~m}$ sub-bottom, respectively. Remarkably, despite the many assumptions implicit in this calculation, the derived depths generally correspond to the depths to the evaporitic unit as estimated from seismic profiles (see Austin et al., this volume).

This approach can also be applied to sites where there is controversy as to whether a buried shallow-water platform exists at all. In particular, at Site 626 in the Florida Straits, which failed to penetrate the primary objective, $\mathrm{Ca}^{2+}$ gradients are similar to 
Table 6 (continued).

\begin{tabular}{|c|c|c|c|c|c|c|c|c|c|c|c|c|}
\hline $\begin{array}{l}\text { Core, section, and } \\
\text { sample interval }(\mathrm{cm})\end{array}$ & $\begin{array}{l}\text { Dolomite } \\
\text { fines } \\
\text { (wt } \% \text { ) }\end{array}$ & $\begin{array}{l}\text { Calcite } \\
\text { fines } \\
(w t \%)\end{array}$ & $\begin{array}{c}\text { Aragonite } \\
\text { fines } \\
(w t \%)\end{array}$ & $\begin{array}{l}\text { Quartz } \\
\text { fines } \\
\text { (wt \%) }\end{array}$ & $\begin{array}{l}\text { Dolomite } \\
\text { coarse } \\
\text { (wt } \% \text { ) }\end{array}$ & $\begin{array}{l}\text { Calcite } \\
\text { coarse } \\
(\text { wt } \%)\end{array}$ & $\begin{array}{l}\text { Aragonite } \\
\text { coarse } \\
\text { (wt } \%)\end{array}$ & $\begin{array}{l}\text { Quartz } \\
\text { coarse } \\
\text { (wt \%) }\end{array}$ & $\begin{array}{l}\text { Fines } \\
(\%)\end{array}$ & $\begin{array}{c}\text { Coarse } \\
(\%)\end{array}$ & $\begin{array}{c}\mathrm{Sr} \\
\text { fines } \\
(\mathrm{ppm})\end{array}$ & $\begin{array}{l}\mathrm{Sr} \\
\text { coarse } \\
\text { (ppm) }\end{array}$ \\
\hline \multicolumn{13}{|l|}{ Hole $630 \mathrm{C}$} \\
\hline $1 \mathrm{H}-1,145-150$ & 0 & 40.2 & 59.0 & 0.80 & 2.1 & 92.0 & 4.50 & 1.3 & 73.41 & 26.59 & 4623 & 1876 \\
\hline $1 \mathrm{H}-2,145-150$ & 0 & 60.4 & 39.6 & 0 & 3.2 & 93.9 & 2.20 & 0.7 & 90.25 & 9.75 & 3212 & 1750 \\
\hline $1 \mathrm{H}-3,145-150$ & 0 & 57.6 & 42.4 & 0 & 17.4 & 80.5 & 1.20 & 0.8 & 84.00 & 16.00 & 2680 & 1381 \\
\hline $1 \mathrm{H}-4,145-150$ & 2.80 & 68.9 & 27.5 & 0.80 & 4.1 & 94.2 & 0.80 & 0.9 & 80.74 & 19.26 & 1900 & 1175 \\
\hline $1 \mathrm{H}-5,145-150$ & 2.40 & 66.7 & 29.4 & 1.50 & 1.9 & 97.1 & 1.00 & 0 & 87.13 & 12.87 & 2275 & 1351 \\
\hline $1 \mathrm{H}-6,145-150$ & 2.30 & 70 & 26.5 & 1.30 & 2.5 & 96.5 & 0.30 & 0.7 & 77.81 & 22.19 & 2267 & 1280 \\
\hline
\end{tabular}

Hole 631A

$1 \mathrm{H}-5,140-150$
$2 \mathrm{H}-5,140-150$
$3 \mathrm{H}-5,140-150$
$4 \mathrm{H}-5,140-150$
$5 \mathrm{H}-5,140-150$
$7 \mathrm{H}-5,140-150$
$10 \mathrm{H}-5,140-150$
$13 \mathrm{X}-5,140-150$
$16 \mathrm{X}-1,140-150$
$19 \mathrm{X}-5,140-150$

$\begin{array}{rl}1.50 & 39.6 \\ 1.70 & 60 \\ 1.90 & 58.5 \\ 3.00 & 47.0 \\ 0 & 56.9 \\ 1.40 & 48.6 \\ 5.90 & 35.0 \\ 14.10 & 32.7 \\ 8.80 & 39.6 \\ 9.20 & 42.0\end{array}$

$\begin{array}{ll}58.9 & 0 \\ 37.4 & 0.90 \\ 39.0 & 0.60 \\ 50 & 0 \\ 41.4 & 1.70 \\ 50 & 0 \\ 59.1 & 0 \\ 53.2 & 0 \\ 51.6 & 0 \\ 48.0 & 0.80\end{array}$

$\begin{array}{rr}0.6 & 31.3 \\ 3.9 & 63.2 \\ 2.1 & 69.5 \\ 2.1 & 54.4 \\ 1.1 & 69.5 \\ 5.9 & 52.8 \\ 8.5 & 39.1 \\ 14.5 & 30.8 \\ 9.0 & 45.0 \\ 5.2 & 50.6\end{array}$

68.10
32.80
27.70
42.40
29.20
40.20
52.40
54.30
45.80
43.00

$\begin{array}{ll}0 & 89.98 \\ 0 & 88.47 \\ 0.7 & 92.31 \\ 1.1 & 90 \\ 0.2 & 79.53 \\ 1.1 & 83.90 \\ 0 & 91.19 \\ 0.4 & 87.05 \\ 0.2 & 81.48 \\ 1.2 & 79.60\end{array}$

$10 \quad 2$
11.53
7.69
10
20.47
16.10
8.81
12.95
18.52
20.40

$\begin{array}{ll}4712 & 5241 \\ 3442 & 2675 \\ 3165 & 2013 \\ 3918 & 3219 \\ 3444 & 2800 \\ 4002 & 3439 \\ 4626 & 3779 \\ 4656 & 4728 \\ 4738 & 4385 \\ 4772 & 3927\end{array}$

Hole 632A

$1 \mathrm{H}-4,140-150$
$2 \mathrm{H}-5,140-150$
$3 \mathrm{H}-4,140-150$
$4 \mathrm{H}-5,140-150$
$5 \mathrm{H}-5,140-150$
$8 \mathrm{H}-5,140-150$
$12 \mathrm{H}-5,140-150$

$\begin{array}{ll}0 & 60 \\ 0 & 24.0 \\ 2.00 & 53.1 \\ 0 & 15.0 \\ 0 & 46.0 \\ 7.70 & 51.5 \\ 5.60 & 65.4\end{array}$

$\begin{array}{ll}37.0 & 3.00 \\ 74.9 & 1.10 \\ 43.5 & 1.40 \\ 85.0 & 0 \\ 54.0 & 0 \\ 40 & 0.70 \\ 27.9 & 1.10\end{array}$

$\begin{array}{lr}2.7 & 59.0 \\ 2.0 & 16.6 \\ 0.9 & 49.5 \\ 4.1 & 8.9 \\ 0.5 & 28.5 \\ 7.8 & 70.1 \\ 4.8 & 81.0\end{array}$

40
81.50
48.80
87.00
71.00
21.30
12.90

$\begin{array}{ll}0.4 & 92.60 \\ 0 & 80.3 \\ 0.9 & 89.5 \\ 0 & 55.56 \\ 0 & 26.37 \\ 0.8 & 83.87 \\ 1.2 & 96.40\end{array}$

$\begin{array}{rrr}7.40 & 3408 & 2396 \\ 19.69 & 5995 & 7050 \\ 10.50 & 3601 & 3545 \\ 44.44 & 7069 & 8371 \\ 73.63 & 3990 & 6194 \\ 16.13 & 3496 & 2668 \\ 3.60 & 2712 & 1725\end{array}$

Hole 632B

10R-2, 140-150

$\begin{array}{ll}5.80 & 67.3 \\ & \\ & \\ 1.40 & 70.3 \\ 0 & 74.0 \\ 2.90 & 59.5 \\ 0 & 76.4 \\ 1.80 & 88.0 \\ 13.00 & 23.0 \\ 7.40 & 46.8 \\ 6.10 & 69.7 \\ 4.30 & 59.0 \\ & \end{array}$

$\begin{array}{ll}25.7 & 1.30 \\ & \\ 27.5 & 1.20 \\ 26.0 & 0 \\ 35.8 & 1.80 \\ 23.6 & 0 \\ 10.1 & 0.10 \\ 64.0 & 1.00 \\ 45.8 & 0 \\ 23.5 & 0.70 \\ 34.5 & 2.10\end{array}$

$\begin{array}{rr}4.8 & 54.5 \\ & \\ 2.0 & 76.6 \\ 4.2 & 95.8 \\ 4.5 & 80.2 \\ 3.0 & 88.0 \\ 3.4 & 96.1 \\ 12.3 & 27.0 \\ 6.9 & 60.1 \\ 2.4 & 94.6 \\ 6.0 & 32.0\end{array}$

40.70

21.50
0
14.00
8.60
0.50
60.70
30.80
3.00
62.00

$\begin{array}{lcrrr}0 & 20.48 & 79.52 & & \\ & & & & \\ 0 & 93.94 & 6.06 & 2759 & 1863 \\ 0 & 93.01 & 6.99 & 2094 & 1287 \\ 1.3 & 92.86 & 7.14 & 3247 & 2066 \\ 0.5 & 89.38 & 10.62 & 2941 & 2313 \\ 0 & 91.76 & 8.24 & 1799 & 1317 \\ 0 & 78.68 & 21.32 & 5117 & 5166 \\ 2.1 & - & - & 3473 & 2472 \\ 0 & - & - & 2362 & 1389 \\ 0 & 40.54 & 59.46 & 3249 & 4229\end{array}$

Hole 634A

$1 \mathrm{H}-5,140-150$

$2 \mathrm{H}-5,140-150$

$3 \mathrm{H}-5,140-150$

$7 \mathrm{H}-5,140-150$

$3 \mathrm{X}-5,140-150$

$6 \mathrm{X}-5,140-150$
$23 \mathrm{X}-3,140-150$

$1 \mathrm{R}-4,140-150$
$2 \mathrm{R}-4,140-150$

$4 \mathrm{R}-2,140-150$

$\begin{array}{ll}6.80 & 89.6 \\ 2.70 & 93.6 \\ 1.50 & 96.2\end{array}$

\begin{tabular}{|c|c|c|c|c|c|c|c|c|c|}
\hline 0 & 3.60 & 8.9 & 89.1 & 0 & 2.1 & 96.30 & 3.70 & - & - \\
\hline 0 & 3.80 & 2.2 & 92.6 & 0 & 5.3 & 91.47 & 8.53 & - & - \\
\hline 0 & 2.30 & 2.0 & 95.0 & 0 & 3.0 & 96.39 & 3.61 & - & - \\
\hline
\end{tabular}

those at Sites 628 and $630(0.024$ to $0.04 \mathrm{mmol} / \mathrm{m})$. This suggests that the evaporitic unit, if indeed it is present below the Straits of Florida, would be at a depth between 600 and $1000 \mathrm{~m}$ sub-bottom, in agreement with results from seismic profiles (Austin et al., this volume) and subsidence studies (Williams et al., this volume). At Site 634 , however, the extremely weak $\mathrm{Ca}^{2+}$ gradient $(0.006 \mathrm{mmol} / \mathrm{m})$ indicates that if a similar $\mathrm{Ca}^{2+}$ source is present, it must be located at approximately $4000 \mathrm{~m}$ sub-bottom. However, owing to the many implicit assumptions within these calculations, this is by no means definitive evidence of the nature of the deposits underlying either area.

\section{CONCLUSIONS}

1. Despite the large depth to basement in the Bahamas region, the alteration of underlying units is an important control on pore-water chemistry at the Little Bahama Bank sites.

2. Aragonite persists to significant depths in the sediment column, indicating that it is more stable in the deep marine diagenetic environment than had been previously supposed.
3. Precipitation of celestite may affect both dissolved $\mathrm{Sr}^{2+}$ and $\mathrm{SO}_{4}^{2-}$ concentrations. Therefore, it may be necessary, to reevaluate the appropriateness of dissolved $\mathrm{Sr}^{2+}$ profiles as tools for assessing the extent of sediment diagenesis.

4. High alkalinity levels favor dolomitization in the Exuma Sound deposits, but the current flux of $\mathrm{Mg}^{2+}$ is insufficient to account for observed dolomite concentrations.

5. Calculated rates of recrystallization in these periplatform deposits are similar to those calculated for deep-sea pelagic carbonate sites.

6. Geochemical profiles support the theory that a shallow carbonate platform of Albian age underlies the area now occupied by the Straits of Florida. However, the geochemical data suggest that this platform did not extend to the area now occupied by Northeast Providence Channel.

\section{ACKNOWLEDGMENTS}

This work was supported in part by a grant from USSAC-JOI. We thank Brad Julson and Tamara Frank for their assistance in the chemis- 
try laboratory. Paul Comet and Art Moore provided much-appreciated friendship and intellectual stimulation during the cruise. Help with the shore-based analyses was provided by the crew of the stable-isotope laboratory. Discussions with P. Baker and P. Stout helped our assessment of the data. J. A. Austin, Jr., P. Baker, J. Gieskes, A. A. Palmer, and W. Schlager reviewed the manuscript.

\section{REFERENCES}

Austin, J. A., Jr., Schlager, W., et al., 1986. Proc. ODP, Init. Repts., 101: College Station, TX (Ocean Drilling Program).

Baker, P. A., 1981. The diagenesis of marine carbonate sediments: experimental and natural observations [Ph.D. dissert.]. Univ. California, San Diego.

1986. Pore-water chemistry of carbonate-rich sediments, Lord Howe Rise, southwest Pacific Ocean. In Kennett, J. P., von der Borch, C. C., et al., Init. Repts. DSDP, 90: Washington (U.S. Govt. Printing Office), 1249-1256.

Baker, P. A., and Bloomer, S. H., 1988. The origin of celestite in deepsea carbonate sediments. Geochim. Cosmochim. Acta, 52:335-340.

Baker, P. A., Gieskes, J. M., and Elderfield, H., 1982. Diagenesis of carbonates in deep-sea sediments-evidence from $\mathrm{Sr} / \mathrm{Ca}$ ratios and interstitial dissolved $\mathrm{Sr}^{2+}$ data. J. Sediment. Petrol., 52:71-82.

Berner, R. A., 1980. Early Diagenesis: a Theoretical Approach: Princeton, NJ (Princeton University Press).

Compton, J. S., and Siever, R., 1986. Diffusion and mass balance of $\mathrm{Mg}$ during early dolomite formation, Monterey Formation. Geochim. Cosmochim. Acta, 50:125-135.

Culberson, C. H., Latham, G., and Bates, R. G., 1978. Solubilities and activity coefficients of calcium and strontium sulfates in synthetic seawater at 0.5 and $25^{\circ} \mathrm{C}$. J. Phys. Chem., 82, 25:2693-2699.

Delaney, M. L., 1983. Foraminiferal trace elements: uptake, diagenesis and 100 m.y. paleochemical history [Ph.D. dissert.]. Mass. Inst. Technol./Woods Hole Oceanogr. Inst.

Droxler, A. W., 1984. Late Quaternary glacial cycles in the Bahamian deep basins and in the adjacent Atlantic Ocean [Ph.D. dissert.]. Univ. Miami.

Dyrssen, D., and Sillen, L. G., 1967. Alkalinity and total carbonate in sea water: A plea for p-T-independent data. Tellus, 19:119-121.

Eaton, M .R., and Boardman, M. R., 1985. North African dust and its relation to paleoclimate recorded in a sediment core from Northwest Providence Channel, Bahamas. Geol. Soc. Am. Abstr. Prog., 17: 572. (Abstract)

Elderfield, H., Gieskes, J. M., Baker, P. A., Oldfield, R. K., Hawkesworth, C. J., and Miller, R., $1982 .{ }^{87} \mathrm{Sr} /{ }^{86} \mathrm{Sr}$ and ${ }^{18} \mathrm{O} /{ }^{16} \mathrm{O}$ ratios, interstitial water chemistry and diagenesis in deep-sea carbonate sediments of the Ontong-Java Plateau. Geochim. Cosmochim. Acta, 46: 2259-2268.

Gieskes, J. M., 1973. Interstitial water studies, Leg 15-alkalinity, pH, $\mathrm{Mg}, \mathrm{Ca}, \mathrm{Si}, \mathrm{PO}_{4}$. In Heezen, B. C., MacGregor, I. D., et al., Init. Repts. DSDP, 20: Washington (U.S. Govt. Printing Office), 813829.

1974. Interstitial water studies, Leg 25. In Simpson, E.S.W., Schlich, R., et al., Init. Repts. DSDP, 25: Washington (U.S. Govt. Printing Office), 361-394.

1981. Deep-sea drilling interstitial water studies: Implications for chemical alteration of the oceanic crust, layers I and II. In Warme, J. E., Douglas, R. G., and Winterer, E. L. (Eds.), The Deep Sea Drilling Project: a Decade of Progress. Soc. Econ. Paleontol. Mineral. Spec. Publ., 32:149-167.

Gieskes, J. M., Elderfield, H., Lawrence, J. R., Johnson, J., Meyers, B., and Campbell, A., 1982. Geochemistry of interstitial waters and sediments, Leg 64, Gulf of California. In Curray, J. R., Moore, D. G., et al., Init. Repts. DSDP, 64: Washington (U.S. Govt. Printing Office), 675-694.
Grasshoff, K., 1976. Methods of Seawater Analysis: New York (Verlag Chemie).

Guzikowski, M., Swart, P. K., and Hamilton, J., 1986. The strontium concentration and isotopic composition of porewaters from Leg 101 of the ODP. Eos, Trans. Am. Geophys. Union, 67:1064. (Abstract)

Katz, A., Sass, E., Starinsky, A., and Holland, H. D., 1972. Strontium behavior in the aragonite-calcite transformation: an experimental study of $40-98^{\circ} \mathrm{C}$. Geochim. Cosmochim. Acta, 36:481-496.

Kennett, J. P., von der Borch, C. C., et al., 1985. Init. Repts. DSDP, 90: Washington (U.S. Govt. Printing Office).

Lawrence, J. R., Gieskes, J. M., and Broecker, W. S., 1975. Oxygen isotope and cation composition of DSDP pore water and the alteration of layer II basalts. Earth Planet. Sci. Lett., 27:1-10.

MacDonald, R. W., and North, N. A., 1974. The effect of pressure on the solubility of $\mathrm{CaCO}_{3}, \mathrm{CaF}_{2}$, and $\mathrm{SrSO}_{4}$ in water. Can. J. Chem., 52:3181-3186.

McDuff, R. E., and Gieskes, J. M., 1976. Calcium and magnesium profiles in DSDP interstitial waters: diffusion or reaction? Earth Planet. Sci. Lett., 33:1-10.

Matter, A., Douglas, R. G., and Perch-Nielsen, K., 1975. Fossil preservation, geochemistry and diagenesis of pelagic carbonates from the Shatsky Rise, northwest Pacific. In Larson, R. L., Moberly, R., et al., Init. Repts. DSDP, 32: Washington (U.S. Govt. Printing Office), 891-921.

Milliman, J. D., Müller, G., and Forstner, U., 1974. Recent Sedimentary Marine Carbonates: New York (Springer-Verlag).

Mullins, H. T., Heath, K. C., Van Buren, M., and Newton, K., 1984. Anatomy of a modern open-oceanic carbonate slope: northern Little Bahama Bank. Sedimentology, 31:141-168.

Reardon, E. J., and Armstrong, D. K., 1987. Celestite $\left(\mathrm{SrSO}_{4(\mathrm{~s})}\right)$ solubility in water, seawater and $\mathrm{NaCl}$ solution. Geochim. Cosmochim. Acta, 51:63-72.

Sayles, F. L., and Mannheim, F. T., 1975. Interstitial solutions and diagenesis in deeply buried marine sediments: Results from the Deep Sea Drilling Project. Geochim. Cosmochim. Acta, 39:103-127.

Schlager, W., and James, N. P., 1978. Low-magnesian calcite limestones forming at the deep-sea floor, Tongue of the Ocean, Bahamas. Sedimentology, 25:675-702.

Schlanger, S. O., and Douglas, R. G., 1974. The pelagic ooze-chalklimestone transition and its implications for marine stratigraphy. In Hsü, K. J., and Jenkyns, H. C. (Eds.), Pelagic Sediments: On Land and Under the Sea: Int. Assoc. Sedimentol. Spec. Publ., 1:117-148.

Schlanger, S. O., Jackson, E. D., et al., 1976. Init. Repts. DSDP, 33: Washington (U.S. Govt. Printing Office).

Simms, M., 1984. Dolomitization by groundwater flow systems in carbonate platforms. Trans. Gulf Coast Assoc. Geol. Soc., 34:411-420.

Stout, P. M., 1985. Interstitial water chemistry and diagenesis of biogenic sediments from the eastern equatorial Pacific, DSDP Leg 85. In Mayer, L., Theyer, F., et al., Init. Repts. DSDP, 85: Washington (U.S. Govt. Printing Office), 805-820.

Swart, P. K., 1981. The strontium, magnesium and sodium composition of recent scleractinian coral skeletons as standards for palaeoenvironmental analyses. Palaeoecol. Palaeogeogr. Palaeoclimatol., 34: 115-136.

Zemmels, I., Cook, H. E., and Hathaway, J. C., 1972. X-ray mineralogy studies-Leg 11. In Hollister, C. D., Ewing, J. I., et al., Init. Repts. DSDP, 11: Washington (U.S. Govt. Printing Office), 729789.

Date of initial receipt: 21 November 1986

Date of acceptance: 23 June 1987

Ms 101B-158 